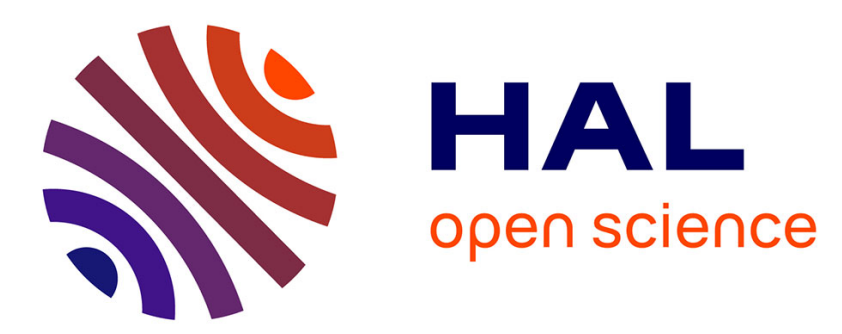

\title{
Double zero bifurcation of non-linear viscoelastic beams under conservative and non-conservative loads
}

\author{
Angelo Luongo, Francesco d'Annibale
}

\section{To cite this version:}

Angelo Luongo, Francesco d'Annibale. Double zero bifurcation of non-linear viscoelastic beams under conservative and non-conservative loads. International Journal of Non-Linear Mechanics, 2013, 55, pp.128-139. hal-00909808

\section{HAL Id: hal-00909808 \\ https://hal.science/hal-00909808}

Submitted on 26 Nov 2013

HAL is a multi-disciplinary open access archive for the deposit and dissemination of scientific research documents, whether they are published or not. The documents may come from teaching and research institutions in France or abroad, or from public or private research centers.
L'archive ouverte pluridisciplinaire HAL, est destinée au dépôt et à la diffusion de documents scientifiques de niveau recherche, publiés ou non, émanant des établissements d'enseignement et de recherche français ou étrangers, des laboratoires publics ou privés. 


\title{
Double zero bifurcation of non-linear viscoelastic beams under conservative and non-conservative loads
}

\author{
A. Luongo*, F. D'Annibale \\ Dipartimento di Ingegneria Civide, Edile-Architettura e Ambientale, University of L'Aquila, 67100 L'Aquilk, Italy
}

\begin{abstract}
A B S T R A C T
The combined effects of conservative and non conservative loads on the mechanical behavior of an unshearable and inextensional visco elastic beam, close to bifurcation, are investigated. The equations of motion and boundary conditions are derived via a constrained variational principle, and the Lagrange multiplier successively condensed, to get integro differential equations. These latter, with the mechanical boundary conditions appended, are put in an operator form, amenable to perturbation analysis. A linear stability analysis is carried out in the space of the two loading parameter, displaying the existence of codimension 1 and codimension 2 bifurcations. The influence of both internal and external damping on this scenario is thoroughly investigated. A post critical analysis is carried out around a double zero bifurcation. by using an adapted version of the multiple scale method, based on fractional series expansions in the perturbation parameter. The integro differential problem is directly attacked, so that any a priori discretization is avoided. Emphasis is given to the interaction between the two damping coefficients. This reveals the existence, also in the non linear range, of a phenomenon of destabilization, so far known only in the linear range.
\end{abstract}

\section{Introduction}

Stability of structures caused by non conservative loads is a classical problem, which involves interesting theoretical aspects $\{1,2\}$. Recently, however, it also took great interest in technical applications. As few examples, the problem is encountered: (a) in aerospace, where tangential forces are produced by jets and rocket motors [3 6]; (b) in piezoelectric controlled systems, where tangential forces are produced by piezoelectric actuators [ 7 14]; (c) in structural and geotechnical engineering [15 22], where tangential or volumic forces are produced by fluid actions [15]. or pore pressure [ [20 22].

In most of these papers, the damping acts on the structural system as an extemal cause, due to aerodynamic effects (see e.g. [23], where both damping forces and couples acting on a tip mass were taken into account). However, damping can also manifest itself as an internal cause, due to dissipation of the material. Comparatively simple linear rheological models, employed in the literature, are the purely viscous and the Kelvin Voigt visco elastic model [24]. The simultaneous presence of internal and external damping has also been considered in [25].

\footnotetext{
* Corresponding author. Tel.: +390862434521.

E-mail addresses: angelo.luongođunivaq, it (A. Luongo), francescadannibale@univaq.it (F. D'Annibale).
}

Damping sometimes produces unpredictable effects on stability. They were first studied by Ziegler [26]. which discovered a phenomenon called "the destabilization paradox". According to this, the loss of stability of a non conservative system with vanishingly small damping manifests itself at a load significantly lower than the critical value relevant to the undamped system. This effect was, subsequently, observed in many non conservative mechanical systems [ 27 32].

A different mechanism of interaction, leading to possibly richer bifurcation scenarios, occurs between non conservative and con servative loads. The topic has been addressed in the linear [33,34] and non linear range, for specific $[35,36]$ or general systems [37,38]. However, it seems that the bifurcation of non linear systems, internally and externally damped, under simultaneous conservative and non conservative loads, has not been investigate, yet. This paper is an attempt to address the problem.

In this paper, linear and non linear bifurcations of beams, under combined conservative and non conservative loads and in the presence of internal and external damping. are analyzed. Preliminary results of this study were presented, in a very short form in [39], where neither no justifications of the model nor of the algorithm was given. In particular, two sample systems considered there, which differ for the damping coefficient values, surprising exhibited completely different postcritical scenarios around a double zero, namely a super or sub critical divergence, the second of which, being more dangerous, caused by larger 
values of damping! Therefore, an apparent destabilization paradox seemed to be found even in the non linear field, this requesting further investigations. The main goal of this paper is therefore to highlight the effects of the damping on the double zero bifurca tion, both on the linear stability and on the postcritical behavior, displaying the mechanism which leads from a non catastrophic to a catastrophic bifurcation.

Several novelties are introduced here, with respect to [39], namely: (1) the equations of motion are derived, and their operator form introduced for algorithmic purposes; (2) the eva luation of the linear stability diagram is analytically justified; (3) the behavior of the eigenvalues close to the double zero bifurcation point is illustrated; (4) the perturbation algorithm employed for post critical analysis is detailed; (5) new results, relevant to the linear stability diagram, are presented, aimed to investigate the role of the damping coefficients; (6) new results, concerning the postcritical scenario, are displayed, with the purpose to illustrate the transition from two different types of bifurcations.

The paper is thus organized. In Section 2 the equations of motions, derived in the Appendix A, are recast in operator form, to include boundary conditions. In Section 3 the eigenvalue problem is addressed, for the linearized system and its adjoint. Here, the linear stability problem is analyzed in the load parameter space and the critical scenario is depicted for various combinations of damping coefficients. In Section 4 a post critical analysis is carried out around a double zero bifurcation point. An adapted version of the multiple scale method (see [40]) is used, which is based on a fractional series expansion of the perturbation parameter, simi larly to the analysis carried out in [41 43]. The bifurcation equations are derived and numerically studied to built up equili brium paths and bifurcation diagrams. In Section 5 some conclu sions are drawn. Two Appendices close the paper.

\section{Model}

The order three equations of motion of a non linear cantilever beam, externally damped and made of a visco elastic material, simultaneously loaded at the tip by a gravitational and a follower force, can be derived via the extended Hamilton's principle (see Appendix A). Internal constraints expressing unshearability and inextensibility of the beam, call for introducing a Lagrange multi plier, which can be eliminated by integration, thus leading to an integro differential problem with relevant geometric (essential) and mechanical (natural) boundary conditions. It is convenient, for algorithmic purposes, to incorporate these latter conditions in the field equations, thus recasting the problem in an operator form, which is amenable to perturbation treatment (see also [41]). When suitable non dimensional quantities are introduced, the equations of motion assume the form

$$
\begin{aligned}
& \mathbf{M} \ddot{\mathbf{u}}+\mathbf{C} \dot{\mathbf{u}}+\mathbf{K u}=\mathbf{n}\left((\mathbf{u}, \dot{\mathbf{u}})^{3}\right) \\
& \mathbf{g u}=\mathbf{0}
\end{aligned}
$$

where

$$
\begin{gathered}
\mathbf{u}:=\left\{\begin{array}{c}
u \\
u_{B} \\
u^{\prime}{ }_{B}
\end{array}\right\}, \quad \mathbf{M}:=\left[\begin{array}{lll}
1 & 0 & 0 \\
0 & 0 & 0 \\
0 & 0 & 0
\end{array}\right], \quad \mathbf{C}:=\left[\begin{array}{ccc}
\beta+\alpha D^{4} & 0 & 0 \\
\alpha D_{B}^{3} & 0 & 0 \\
\alpha D_{B}^{2} & 0 & 0
\end{array}\right] \\
\mathbf{g u}:=\left\{\begin{array}{c}
u_{A} \\
u_{A}^{\prime}
\end{array}\right\}, \quad \mathbf{K}:=\left[\begin{array}{cccc}
D^{4}+2(\mu+v) D^{2} & 0 & 0 \\
D_{B}^{3} 2 v D_{B}^{1} & 0 & 0 \\
D_{B}^{2} & 0 & 0
\end{array}\right]
\end{gathered}
$$

$$
\mathbf{n}\left((\mathbf{u}, \dot{\mathbf{u}})^{3}\right):=\left\{\begin{array}{c}
{\left[u^{\prime}\left(u^{\prime} u^{\prime \prime}\right)^{\prime}\right]^{\prime}+\mu u_{B}^{\prime 2} u^{\prime \prime}} \\
\alpha\left\{\left[u^{\prime}\left(u^{\prime} u^{\prime \prime}\right)^{\prime}\right]^{\prime}\right\}^{\bullet} \\
\frac{1}{2}\left[\left(\int_{1}^{s}\left(\int_{0}^{s} u^{\prime 2} d s\right)^{\bullet \bullet} d s\right) u^{\prime}\right]^{\prime} \\
u_{B}^{\prime \prime \prime} u_{B}^{\prime 2}+u_{B}^{\prime \prime} u_{B}^{\prime} \\
\mu u_{B}^{\prime 2} u_{B}^{\prime}+\alpha\left(u_{B}^{\prime \prime} u_{B}^{\prime 2}+u^{\prime \prime 2} u_{B}^{\prime}\right)^{\bullet} \\
u_{B}^{\prime \prime} u_{B}^{\prime 2} \alpha\left(u_{B}^{\prime \prime} u_{B}^{\prime 2}\right)^{\bullet}
\end{array}\right\}
$$

together with the clamp conditions $u_{A}=0, u_{A}^{\prime}=0$. Here, $u(s, t)$ is the transverse displacement at the abscissa $s$ and time $t, \mu$ is the follower force, $\nu$ is the gravitational force, $\alpha$ is the internal damping, $\beta$ is the external damping, $A$ and $B$ denote the ends of the beam, a dash $s$ differentiation and a dot $t$ differentiation. Moreover, $\mathbf{M}, \mathbf{C}$ and $\mathbf{K}$ are the mass, damping and stiffness operators, respectively, acting on the vector $\mathbf{u} \in \tilde{\mathbb{T}}$, with $\tilde{\square}:=\mathbb{\llbracket} \oplus \mathbb{R}^{2}$, which collects the field displacement $u$ and the displacement and its spatial derivative evaluated at the end $B ; \mathbf{n}\left((\mathbf{u}, \dot{\mathbf{u}})^{3}\right)$ is the vector of non linearities (which are cubic homogeneous forms in their arguments and their spatial derivatives and integrals), both in the domain and at the boundary $B$; gu is the vector of the constrained displacements at the end $A$; moreover, $D^{n}:=\partial^{n} / \partial s^{n}, D_{B}^{n}:=\partial^{n} /\left.\partial s^{n}\right|_{B}$. Eqs. (1) are then rewritten in the following state form:

$$
\begin{aligned}
& \mathbf{B} \dot{\mathbf{U}}=\mathbf{A} \mathbf{U}+\mathbf{N}\left(\mathbf{U}^{3}\right) \\
& \mathbf{G U}=\mathbf{0}
\end{aligned}
$$

where

$$
\begin{gathered}
\mathbf{U}:=\left\{\begin{array}{l}
\mathbf{u} \\
\mathbf{v}
\end{array}\right\}, \quad \mathbf{v}:=\left\{\begin{array}{c}
\dot{u} \\
\dot{u}_{B} \\
\dot{u}_{B}^{\prime}
\end{array}\right\}, \quad \mathbf{B}:=\left[\begin{array}{cc}
\mathbf{I} & \mathbf{0} \\
\mathbf{0} & \mathbf{M}
\end{array}\right], \quad \mathbf{G U}:=\left\{\begin{array}{l}
u_{A} \\
u_{A}^{\prime}
\end{array}\right\} \\
\mathbf{A}:=\left[\begin{array}{cc}
\mathbf{0} & \mathbf{I} \\
\mathbf{K} & \mathbf{C}
\end{array}\right], \quad \mathbf{N}\left(\mathbf{U}^{3}\right):=\left\{\begin{array}{c}
\mathbf{0} \\
\mathbf{n}\left(\mathbf{U}^{3}\right)
\end{array}\right\}
\end{gathered}
$$

in which $\mathbf{v}:=\dot{\mathbf{u}}$ is the velocity field, and $\mathbf{U} \in \tilde{\mathbb{T}}^{2}$ is the state vector.

The equations obtained in this section have very similar structure to those showed in [14,44 47]. In all aforementioned papers, controlling system has an independent kinematics, char acterized by a field which is parallel to the mechanical transversal displacements, while being an electrical quantity. It is authors' opinion that the study of buckling of aforementioned piezo electro mechanical structures is feasible with the method presented here.

\section{Eigenvalue analysis}

Bifurcation analysis calls for evaluation of the (right) eigenva lues and eigenvectors of the equations of motion (3). Since the problem is not self adjoint, also the (left) eigenvectors of the adjoint problem must be determined. The procedure, detailed in [41], is here briefly resumed.

\subsection{Right and left eigenvalue problems}

The equations of motion (3), when linearized, admit the solution $\mathbf{U}=\boldsymbol{\Phi} e^{\lambda t}$ which leads to the differential eigenvalue pro blem

$(\mathbf{A} \lambda \mathbf{B}) \boldsymbol{\Phi}=\mathbf{0}$
$\mathbf{G} \boldsymbol{\Phi}=\mathbf{0}$

or, equivalently

$$
\begin{aligned}
& \hat{\boldsymbol{\phi}}=\lambda \boldsymbol{\phi} \\
& \mathbf{K} \boldsymbol{\phi}+\mathbf{C} \hat{\boldsymbol{\phi}}+\lambda \mathbf{M} \hat{\boldsymbol{\phi}}=\mathbf{0} \\
& \phi_{A}=0, \quad \phi^{\prime}{ }_{A}=0
\end{aligned}
$$


having set $\boldsymbol{\Phi}:=\{\boldsymbol{\phi}, \hat{\boldsymbol{\phi}}\}^{\mathrm{T}} \in \tilde{\mathbb{M}}^{2}, \boldsymbol{\phi}:=\left\{\phi, \phi_{B}, \phi^{\prime}{ }_{B}\right\}^{\mathrm{T}} \in \tilde{\mathbb{M}}$ and $\hat{\boldsymbol{\phi}}:=\left\{\hat{\boldsymbol{\phi}}, \hat{\boldsymbol{\phi}}_{B}, \hat{\phi}^{\prime}{ }_{B}\right\}^{\mathrm{T}} \in \tilde{\mathbb{M}}$. By introducing the scalar products in $\tilde{\widetilde{T}}$ and $\tilde{\mathbb{T}}^{2}$, respectively

$(\boldsymbol{\phi}, \boldsymbol{\psi}):=\int_{0}^{1} \bar{\phi}_{1}(s) \psi_{1}(s) d s+\sum_{j} \bar{\phi}_{j, 3} \psi_{j}, \quad \boldsymbol{\phi}, \boldsymbol{\psi} \in \tilde{\mathbb{\complement}}$

$\langle\boldsymbol{\Phi}, \boldsymbol{\Psi}\rangle:=\int_{0}^{1} \sum_{i} \overline{\boldsymbol{\Phi}}_{i, 4}(s) \boldsymbol{\Psi}_{i}(s) d s+\sum_{j} \sum_{2,3,5,6} \bar{\Phi}_{j} \Psi_{j}, \quad \boldsymbol{\Phi}, \boldsymbol{\Psi} \in \tilde{\mathbb{\boxplus}}^{2}$

and using the bilinear identity

$\langle\boldsymbol{\Psi},(\mathbf{A} \lambda \mathbf{B}) \boldsymbol{\Phi}\rangle=\left\langle\left(\mathbf{A}^{*} \bar{\lambda} \mathbf{B}^{*}\right) \boldsymbol{\Psi}, \boldsymbol{\Phi}\right\rangle$

the adjoint eigenvalue problem follows:

$\left(\mathbf{A}^{*} \bar{\lambda} \mathbf{B}^{*}\right) \boldsymbol{\Psi}=\mathbf{0}$

$\mathbf{G}^{*} \boldsymbol{\Psi}=\mathbf{0}$

also written as

$\mathbf{K}^{*} \boldsymbol{\psi}+\bar{\lambda} \hat{\boldsymbol{\psi}}=\mathbf{0}$

$\hat{\boldsymbol{\psi}} \quad \mathbf{C}^{*} \boldsymbol{\psi} \bar{\lambda} \mathbf{M}^{*} \boldsymbol{\psi}=\mathbf{0}$

$\psi_{A}=0, \quad \psi_{A}^{\prime}=0$

in which

$\mathbf{B}^{*}:=\left[\begin{array}{cc}\mathbf{I} & \mathbf{0} \\ \mathbf{0} & \mathbf{M}^{*}\end{array}\right], \quad \mathbf{A}^{*}:=\left[\begin{array}{cc}\mathbf{0} & \mathbf{K}^{*} \\ \mathbf{I} & \mathbf{C}^{*}\end{array}\right]$

$\mathbf{G}^{*} \Psi:=\left\{\begin{array}{c}\psi_{A} \\ \psi^{\prime}{ }_{A}\end{array}\right\}, \quad \mathbf{M}^{*}:=\left[\begin{array}{lll}1 & 0 & 0 \\ 0 & 0 & 0 \\ 0 & 0 & 0\end{array}\right]=\mathbf{M}$

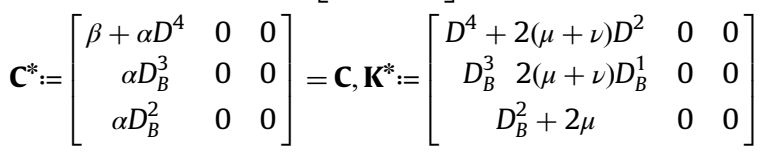

and $\boldsymbol{\Psi}:=\{\hat{\boldsymbol{\psi}}, \boldsymbol{\psi}\}^{\mathrm{T}}$. It should be noticed that while $\mathbf{M}$ and $\mathbf{C}$ are self adjoint, $\mathbf{K}$ is not self adjoint, because of the presence of the follower force at the boundary.

\subsection{Generalized eigenvectors associated with a double zero} eigenvalue

The case in which $\lambda=0$ is a double eigenvalue for Eq. (5) (and (9)) is of particular interest in bifurcation analysis (Takens Bogda nov bifurcation). In this occurrence, there exist only one proper right eigenvector $\boldsymbol{\Phi}_{1}=\left\{\boldsymbol{\phi}_{1}, \mathbf{0}\right\}^{\mathrm{T}}$ and only one proper left eigenvec tor $\boldsymbol{\Psi}_{2}=\left\{\mathbf{C}^{*} \boldsymbol{\psi}_{2}, \boldsymbol{\psi}_{2}\right\}^{\mathrm{T}}$; moreover, they are mutually orthogonal, i.e. $\left\langle\boldsymbol{\Psi}_{2}, \boldsymbol{\Phi}_{1}\right\rangle=\left(\mathbf{C}^{*} \boldsymbol{\psi}_{2}, \boldsymbol{\phi}_{1}\right)=0$. To complete the right basis, a generalized eigenvector $\boldsymbol{\Phi}_{2}=\left\{\boldsymbol{\phi}_{2}, \hat{\boldsymbol{\phi}}_{2}\right\}^{\mathrm{T}}$ is needed, which is solution to

$\mathbf{A} \Phi_{2}=\boldsymbol{\Phi}_{1}$

$\mathbf{G} \boldsymbol{\Phi}_{2}=\mathbf{0}$

or, equivalently

$\hat{\phi}_{2}=\phi_{1}$

$\mathbf{K} \phi_{2} \quad \mathbf{C} \hat{\boldsymbol{\phi}}_{2}=\mathbf{0}$

$\phi_{2 A}=0, \quad \phi_{2 A}^{\prime}=0$

The generalized eigenvector $\boldsymbol{\Phi}_{2}$ is not unique, since $\mathbf{A}$ is singular. To select it univocally, a normalization condition must be enforced, e.g. $\phi_{2 B}=0$; once $\boldsymbol{\Phi}_{2}$ has been normalized, also $\boldsymbol{\Psi}_{2}$ is normalized, by requiring $\left\langle\Psi_{2}, \boldsymbol{\Phi}_{2}\right\rangle=1$.

\subsection{Linear stability analysis}

Stability of the trivial, rectilinear configuration of the beam is governed by the linear eigenvalue problem (1). In extended form, it reads

$(1+\alpha \lambda) \phi^{\mathrm{IV}}+2(\mu+\nu) \phi^{\prime \prime}+\left(\lambda^{2}+\beta \lambda\right) \phi=0$
$\phi_{A}=0, \quad \phi^{\prime}{ }_{A}=0$

$$
(1+\alpha \lambda) \phi^{\prime \prime \prime}{ }_{B} \quad 2 \nu \phi_{B}^{\prime}=0, \quad(1+\alpha \lambda) \phi^{\prime \prime}{ }_{B}=0
$$

The field equation (141) and the boundary conditions at $A$ lead to the solution

$\phi(s)=c_{1}[\cos (p s) \cosh (q s)]+c_{2}\left[\frac{1}{p} \sin (p s) \frac{1}{q} \sinh (q s)\right]$

where

$q^{2}:=\frac{\sqrt{(\mu+\nu)^{2}}(1+\alpha \lambda)\left(\beta \lambda+\lambda^{2}\right) \quad(\mu+\nu)}{(1+\alpha \lambda)}$

$p^{2}:=\frac{\sqrt{(\mu+\nu)^{2}}(1+\alpha \lambda)\left(\beta \lambda+\lambda^{2}\right)+(\mu+\nu)}{(1+\alpha \lambda)}$

and $\mathbf{c}=\left(c_{1}, c_{2}\right)^{\mathrm{T}}$ are arbitrary constants. It should be noted that factors $1 / p, 1 / q$ have been introduced in Eq. (15), in order it holds even when $p \rightarrow 0$ or $q \rightarrow 0$. By enforcing boundary conditions at $B$, two algebraic equations follow:

$\mathbf{S}_{\lambda} \mathbf{c}=\mathbf{0}$

where

$\mathbf{S}_{\lambda}:=\left[\begin{array}{cc}\left(p^{3}+p^{3} \alpha \lambda\right) \sin (p) & \left(p^{2}+p^{2} \alpha \lambda\right) \cos (p) \\ +2 p \nu \sin (p) & 2 \nu \cos (p) \\ +\left(q^{3}+q^{3} \alpha \lambda\right) \sinh (p) & +\left(q^{2}+q^{2} \alpha \lambda\right) \cosh (q) \\ +2 q \nu \sinh (p) & +2 \nu \cosh (q) \\ (1+\alpha \lambda)\left(p^{2} \cos (p)\right) & (1+\alpha \lambda)(p \sin (p)) \\ (1+\alpha \lambda)\left(q^{2} \cosh (q)\right) & (1+\alpha \lambda)(q \sinh (q))\end{array}\right]$

is the "dynamic stiffness matrix" of the system, depending on the eigenvalue $\lambda$. This matrix, however, also depends on the control parameters, $(\mu, \nu)$ and the auxiliary parameters $(\alpha, \beta)$, i.e. $\mathbf{S}_{\lambda}=\mathbf{S}_{\lambda}(\mu, \nu ; \alpha, \beta)$. The characteristic equation $\operatorname{det} \mathbf{S}_{\lambda}(\mu, \nu ; \alpha, \beta)=0$ supplies the eigenvalues $\lambda$ as a function of $(\mu, \nu ; \alpha, \beta)$. To restate the problem in real variables, the eigenvalues are written as $\lambda=\xi+i \omega$, with $\xi, \omega \in \mathbb{R}$, and then the characteristic equation is re written in the form

$f(\xi, \omega ; \mu, \nu ; \alpha, \beta)+i g(\xi, \omega ; \mu, \nu ; \alpha, \beta)=0$

with $f, g \in \mathbb{R}$. For a fixed set of parameter $(\mu, \nu ; \alpha, \beta)^{\mathrm{T}}$, the system of two real equations $f=0, g=0$ furnishes the unknowns $\xi, \omega$. From a geometrical point of view, for given values of the parameters $(\mu, \nu ; \alpha, \beta)$, each of these equations describes a curve in the $(\xi, \omega)$ plane, which intersect each other in an infinite number of points, representing the eigenvalues of the systems.

In order to find the divergence boundary in the control para meter space, the loci $\mathcal{D}$ of the roots $\xi=0, \omega=0$ must be found. Since $g(0,0 ; \mu, \nu ; \alpha, \beta)$ is found to vanish identically for any $(\mu, \nu ; \alpha, \beta)$, and $f(0,0 ; \mu, \nu ; \alpha, \beta)$ is found to be independent of the damping coefficients $\alpha, \beta$, Eq. (19) assumes the following simple form:

$(\mu+\nu)^{3 / 2}[\mu+\nu \cos (\sqrt{ } 2(\mu+\nu))]=0$

This equation implicitly defines a multi branch curve $\mathcal{D}$ on the $(\nu, \mu)$ plane.

Hopf bifurcation occurs at the manifold $\mathcal{H}$ of the parameter plane on which $\xi=0, \omega \neq 0$, defined by

$\left\{\begin{array}{l}f(0, \omega ; \mu, \nu ; \alpha, \beta)=0 \\ g(0, \omega ; \mu, \nu ; \alpha, \beta)=0\end{array}\right.$

These equations, for a given pair of damping coefficients $(\alpha, \beta)$, differently from zero, implicitly define a multi branch curve $\mathcal{H}$ in the $(\nu, \mu)$ plane, parametrized by the $\omega$ parameter. No closed form solutions, but only numerical, can be pursued for Eqs. (21) (see [48]). 


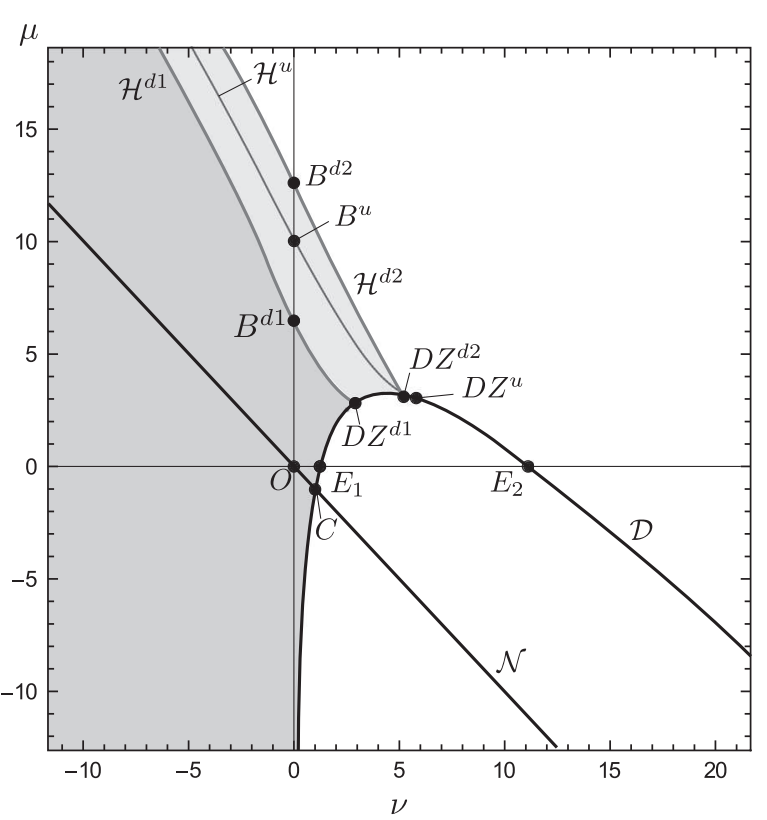

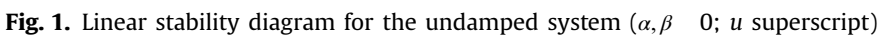

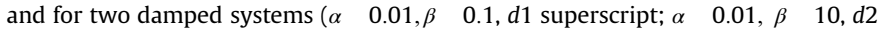
superscript); $\mathcal{D}$ : divergence locus, $\mathcal{H}$ : Hopf locus, $\mathcal{N}$ : zero-stress locus; $E$ : Eulerian bifurcations; $B$ : Beck's bifurcations; $D Z$ : double-zero bifurcations.

When the damping coefficients $(\alpha, \beta)$ are equated to zero in Eq. (14), a circulatory system results, for which all the eigenvalues lie on the imaginary axis in the pre critical phase. In this case, a Hopf bifurcation occurs at the manifold $\mathcal{H}$ of the parameter plane on which two simple eigenvalues merge into a double eigenvalue, that cannot more be defined by system (21), since the $g$ function vanishes, namely $g(0, \omega ; \mu, \nu ; 0,0)=0$. Hopf bifurcation manifold, when this coalescence mechanism takes place, is then defined by

$\left\{\begin{array}{l}f(0, \omega ; \mu, \nu ; 0,0)=0 \\ \frac{\partial f}{\partial \omega}(0, \omega ; \mu, \nu ; 0,0)=0\end{array}\right.$

Again, no closed form solutions, but only numerical, can be pursued for Eqs. (22).

\subsection{Parametric analysis}

A large view of the linear stability diagram, in the $(\nu, \mu)$ plane, has been displayed in [39]. Here, attention is focused, in Fig. 1, on a small region of the same diagram, in which the analysis is carried out. The gray zone denotes stable systems and the white zone denotes unstable systems.

Eq. (20) (divergence locus) defines a family of curves (inde pendent of damping) labeled with $\mathcal{D}$. Eq. (20) also defines an additional straight line $\mathcal{N}$, of equation $\mu=-\nu$, but, as it will be shown ahead, this is not a bifurcation locus, since the transvers ality condition of the eigenvalues is not satisfied on it. Eqs. (22) (Hopf locus) define a second family of curves labeled with $\mathcal{H}^{u}$. Loci $\mathcal{D}$ and $\mathcal{H}$ represent codimension 1 bifurcations. The divergence locus $\mathcal{D}$ intersects the $\nu$ axis at points $E_{1}, E_{2}, \ldots$, each corresponding to an Eulerian critical load, $\nu_{E_{1}}=\pi^{2} / 8, \nu_{E_{2}}=9 \pi^{2} / 8, \ldots$. The Hopf locus $\mathcal{H}^{u}$ intersects the $\mu$ axis at the Beck's points. Only the lower intersection is depicted in Fig. $1, B^{u}:=(0,10.02)$. The Hopf curves die at intersections with the divergence curves, where they merge with the same tangent; in Fig. 1 only the first intersection between the Hopf and divergence family of curves is shown, so codimension 2 bifurcation occurs at point $D Z^{u}:=(5.51,3.02)$.

The straight line $\mathcal{N}$ represents a one parameter family of beams unstressed in the undeformed configuration, since the two forces balance themselves when aligned. However, when the beam moves to an adjacent configuration, and due to the different behavior of the two forces, a (non dimensional) trans versal force proportional to the tip rotation rises, namely $V_{B}:=-\mu u_{B}^{\prime}$. Since $u_{B}$ and $u_{B}^{\prime}$ are concordant in sign in the resulting static deflection, (a) when $\mu>0$ (compressive follower force) $V_{B}$ is opposite to $u_{B}$, and therefore is stabilizing; (b) when $\mu<0$ (tensile follower force), the force is concordant with $u_{B}$, and therefore is (potentially) destabilizing. However, for values of $|\mu|<1$ (corre sponding to the $\overline{O C}$ segment in the figure), $V_{B}$ is smaller than the elastic forces necessary to keep the beam in the deformed configuration, so that the trivial equilibrium is still stable; the opposite occurs for $|\mu|>1$ (below point $C$ ), so that the trivial
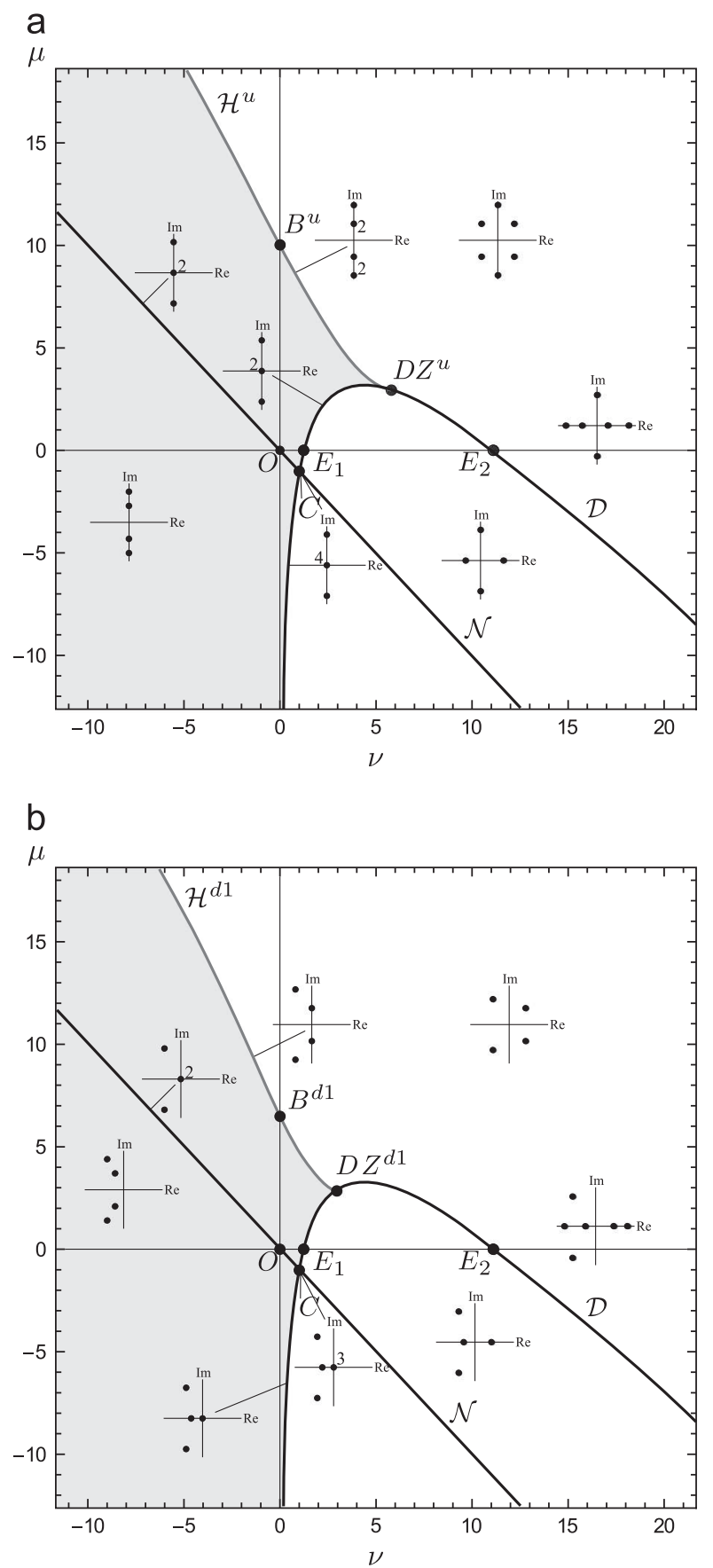

Fig. 2. Linear stability diagrams and eigenvalue sketches: (a) undamped system

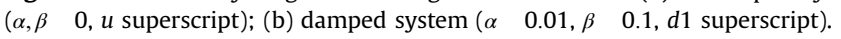


equilibrium is unstable. Therefore $C:=(1,-1)$ is a critical point for this family of systems.

Moreover, in Fig. 1, also the linear stability diagram for two differently damped beams, is depicted and the stable zones of the $(\nu, \mu)$ plane, in both damping cases, are again denoted in gray in the figure. Eqs. (21) (Hopf locus) define a family of curves labeled with $\mathcal{H}$ (depending on damping). Of this family, the curves relevant to the slightly damped sample system $(\alpha=0.01, \beta=0.1)$ have been denoted by $\mathcal{H}^{d 1}$, and curves relevant to a strongly external damped system, $(\alpha=0.01, \beta=10)$ by $\mathcal{H}^{d 2}$; the $\mathcal{H}^{u}$ curve, relevant to the undamped system, is also reported for comparison. The Beck's points for the two damped systems are $B^{d 1}:=(0,6.46)$ and $B^{d 2}:=(0,12.60)$, respectively, one lower and the other higher with respect to the undamped Beck's point. The Hopf curves die at intersections with the divergence curves, according to the well known mechanism of the double zero (or Takens Bogdanov) bifurcation (see for example [49]). Such codimension 2 bifurcation occurs at points $D Z^{d 1}:=(2.88,2.81), D Z^{d 2}:=(5.30,3.07)$, and in both cases, the two loci cross each other transversally.

To investigate the behavior of the eigenvalues in the transition across the boundaries, the stability diagram of Fig. 1 is replicated in Fig. 2, together with the sketches of the eigenvalues relevant to each region. Fig. 2a,b describes the scenario for the undamped ( $u$ superscript) and the slightly damped ( $d 1$ superscript) cases, respectively, and give a sketch of the eigenvalues in the different zones. The undamped case is analyzed first (Fig. 2a). Here, the system is (not asymptotically) stable if all its eigenvalues lie on the imaginary axis, as it occurs in the gray zone. The transition through the divergence curve, moving from the left, occurs as follows: a couple of imaginary eigenvalues first collides at zero (on $\mathcal{D})$ and then split into a couple of opposite in sign real eigenvalues. The transition through the Hopf curve happens as follows: two couples of purely imaginary eigenvalues moves towards each other, coalesce in double eigenvalues (on $\mathcal{H}^{u}$ ), and then splits again into two couples of complex and conjugate eigenvalues, having opposite real parts. The straight line $\mathcal{N}$, instead, does not affect stability. Indeed, by crossing them in the stable zone, a couple of purely imaginary eigenvalues collides at the origin (on $\mathcal{N}$ ), then splits again into two purely imaginary eigenvalues. Therefore, no zero eigenvalue passes through the imaginary axis, and therefore no bifurcations occurs. Similarly, by crossing the line in the unstable zone, a couple of opposite in sign real eigenvalues collides at zero (on $\mathcal{N}$ ), then it splits again into opposite in sign real eigenvalues. At the critical point $C$, a more degenerate condition occurs, where four eigenvalues coalesce at zero. The eigenvalues of the unstable region, further to the right, are also sketched for low and high values of $\mu$.

When the system is damped (Fig. 2b), (asymptotic) stability requires that all the eigenvalues have negative real parts, while the system is unstable if at least one eigenvalue has positive real part. Loss of stability through divergence and Hopf loci occurs via the classical mechanisms, namely: (a) two stable complex conjugate eigenvalues first collide on the real axis, then, one of them crosses the imaginary axis at zero (on $\mathcal{D}$ ); (b) a couple of stable complex conjugate eigenvalues simultaneously crosses the imaginary axis (on $\mathcal{H}^{d 1}$ ). Again, the $\mathcal{N}$ line does not influence stability, since the mechanism is identical to that of the undamped case. At the critical point $C$, three eigenvalues coalesce at zero. Eigenvalues in the rightmost region are also indicated.

A parametric study on the influence of both internal $(\alpha)$ and external $(\beta)$ damping coefficients is then carried out. First, the effect of a single parameter is studied in Fig. 3a,b. In Fig. 3a the $\alpha$ coefficient is zeroed, while the $\beta$ coefficient is varied in a wide range. It is found that, for any value of $\beta$, all the Hopf loci a

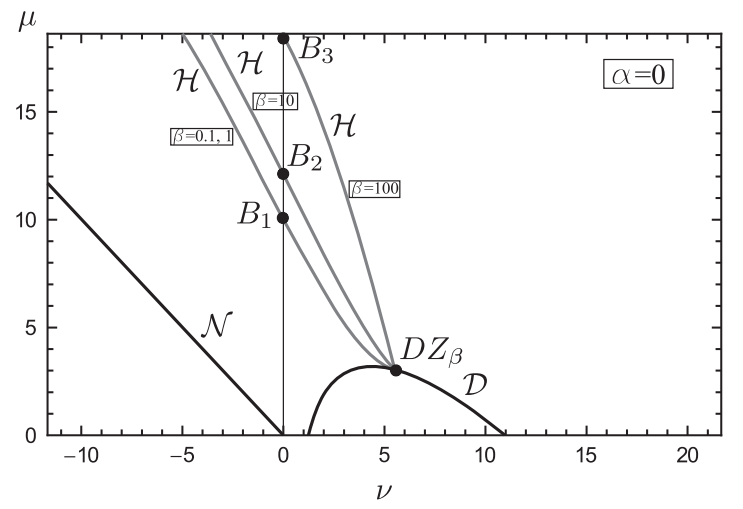

b

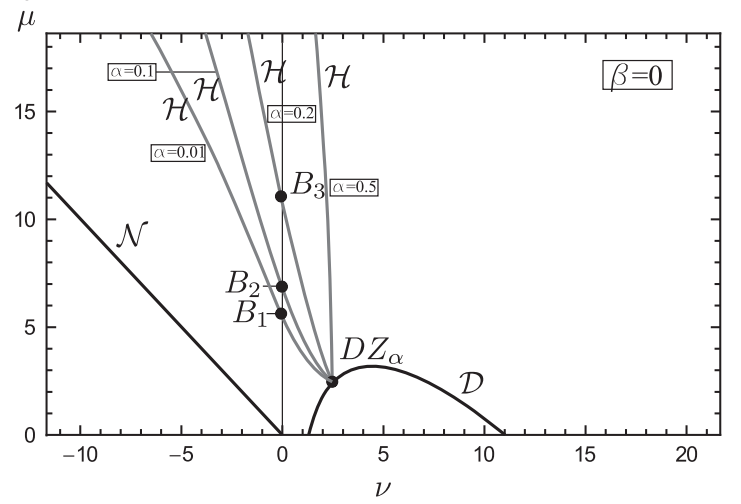

C

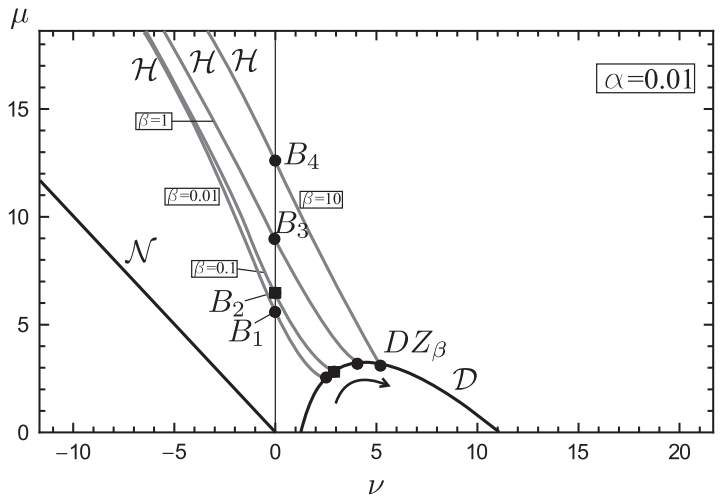

d

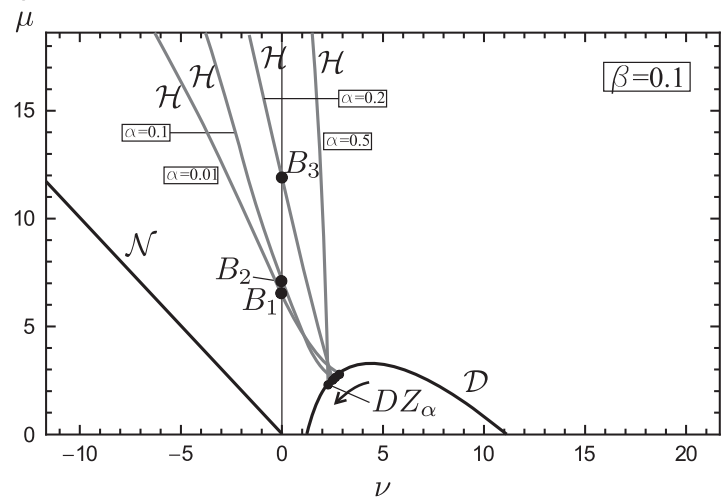

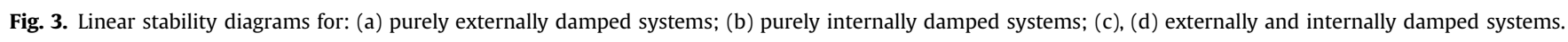


originate from the same double zero point of the undamped system $D Z_{\beta} \equiv D Z^{u}=(5.51,3.02)$. However, curves modify their shape; in particular, the attack angle with the divergence curve increases from zero for increasing $\beta$. As a consequence, the Beck's load also increases with $\beta$ (e.g. $\mu_{B}=10.05,12.13,18.60$ when $\beta=0.01,10,100$, respectively). In Fig. $3 \mathrm{~b} \beta$ is zeroed, while $\alpha$ is varied. Again, the double zero point $D Z_{\alpha}:=(2.46,2.46)$ is found to be independent of the damping value, but it does not coincide with that of the undamped system. Moreover, when $\alpha \rightarrow 0$, the angle of attack to the divergence curve tends to $\pi / 2$, instead of zero. Due to the deformation of $\mathcal{H}$, the Beck's load highly increases with $\alpha$ (e.g. $\mu_{B}=5.48,6.82,10.75$ when $\alpha=0.01,0.1,0.2$, respectively).

To analyze the combined effect of the two parameters, one is kept constant and non zero, and the other is varied (Fig. 3c,d). For fixed $\alpha=0.01$ and increasing $\beta$, the scenario of Fig. 3c is found. The Hopf locus moves to the right, and the double zero point tends to $D Z_{\beta}$. Consequently, the Beck's load increases with $\beta$ (e.g. $\mu_{B}=5.61,6.46,8.90,12.60$, when $\beta=0.01,0.1,1,10$, respectively). For fixed $\beta=0.1$ and increasing $\alpha$ (Fig. 3d), the Hopf locus moves to the right and rotates, intersecting the divergence locus at double zero points tending to $D Z_{\alpha}$. Accordingly, the Beck's load increases with $\alpha$ (e.g. $\mu_{B}=6.46,7.04,10.99$, when $\alpha=0.01,0.1,0.2$ ).

\section{Bifurcation analysis around a double-zero point}

\subsection{Bifurcation equation}

A perturbation parameter $0 \leq \varepsilon \ll 1$, which is a measure of the response amplitude, is introduced via the rescaling $\mathbf{U}(s, t)=\varepsilon^{1 / 2} \hat{\mathbf{U}}(s, t)$, where $\mathrm{O}(\|\hat{\mathbf{U}}(s, t)\|)=1$. Increments $\varepsilon(\hat{\mu}, \hat{\nu})$ of the bifurcation parameters $(\mu, \nu)$ with respect to their bifurcation values $\left(\mu_{0}, \nu_{0}\right)$ are introduced, i.e. $\mu=\mu_{0}+\varepsilon \hat{\mu}, \nu=\nu_{0}+\varepsilon \hat{\nu}$, where $\mathrm{O}(\hat{\mu})=\mathrm{O}(\hat{\nu})=1$. Consequently

$\mathbf{K}=\mathbf{K}_{0}+\varepsilon\left(\hat{\mu} \mathbf{K}_{\mu}+\hat{\nu} \mathbf{K}_{\nu}\right)+\mathrm{O}\left(\varepsilon^{2} \mathbf{I}\right)$

where $\mathbf{K}_{\mu}, \mathbf{K}_{\nu}$ are the derivatives of $\mathbf{K}$ with respect to the parameters. Hats will be omitted ahead for notational conveni ence. Several independent time scales are defined, namely $t_{k}:=\varepsilon^{k / 2} t, \quad(k=0,1, \ldots)$, so that $\mathrm{d} / \mathrm{d} t=\mathrm{d}_{0}+\varepsilon^{1 / 2} \mathrm{~d}_{1}+\varepsilon \mathrm{d}_{2}+$. The unknowns are expanded in series of fractional powers of $\varepsilon$ as

$\mathbf{U}=\sum_{k} \varepsilon^{k / 2}\left\{\begin{array}{l}\mathbf{u}_{k} \\ \mathbf{v}_{k}\end{array}\right\}$

After substitution of the previous expansions in Eqs. (3) a chain of linear perturbation equations and relevant boundary conditions follow (hats omitted):

$$
\begin{aligned}
& \varepsilon^{0}:\left\{\begin{array}{l}
\mathrm{d}_{0} \mathbf{u}_{0} \quad \mathbf{v}_{0}=\mathbf{0} \\
\mathbf{M d}_{0} \mathbf{v}_{0}+\mathbf{K}_{0} \mathbf{u}_{0}+\mathbf{C} \mathbf{v}_{0}=\mathbf{0} \\
u_{0 A}=0, \quad u_{0 A}^{\prime}=0
\end{array}\right. \\
& \varepsilon^{1 / 2}:\left\{\begin{array}{l}
\mathrm{d}_{0} \mathbf{u}_{1} \mathbf{v}_{1}=\mathrm{d}_{1} \mathbf{u}_{0} \\
\mathbf{M d}_{0} \mathbf{v}_{1}+\mathbf{K}_{0} \mathbf{u}_{1}+\mathbf{C} \mathbf{v}_{1}=\mathbf{M d}_{1} \mathbf{v}_{0} \\
u_{1 A}=0, \quad u_{1 A}^{\prime}=0
\end{array}\right. \\
& \varepsilon:\left\{\begin{array}{l}
\mathrm{d}_{0} \mathbf{u}_{2} \quad \mathbf{v}_{2}=\mathrm{d}_{2} \mathbf{u}_{0} \quad \mathrm{~d}_{1} \mathbf{u}_{1} \\
\mathbf{M d}_{0} \mathbf{v}_{2}+\mathbf{K}_{0} \mathbf{u}_{2}+\mathbf{C} \mathbf{v}_{2}=\mathbf{M d}_{2} \mathbf{v}_{0} \\
\mathbf{M d}_{1} \mathbf{v}_{1} \quad\left(\mu \mathbf{K}_{\mu}+\nu \mathbf{K}_{\nu}\right) \mathbf{u}_{0}+\mathbf{n}\left(\mathbf{U}_{0}^{3}\right) \\
u_{2 A}=0, \quad u_{2 A}^{\prime}=0
\end{array}\right. \\
& \varepsilon^{3 / 2}:\left\{\begin{array}{l}
\mathrm{d}_{0} \mathbf{u}_{3} \quad \mathbf{v}_{3}=\mathrm{d}_{3} \mathbf{u}_{0} \quad \mathrm{~d}_{2} \mathbf{u}_{1} \quad \mathrm{~d}_{1} \mathbf{u}_{2} \\
\mathbf{M d}_{0} \mathbf{v}_{3}+\mathbf{K}_{0} \mathbf{u}_{3}+\mathbf{C} \mathbf{v}_{3}=\mathbf{M} \mathbf{d}_{3} \mathbf{v}_{0} \\
\mathbf{M d}_{2} \mathbf{v}_{1} \quad \mathbf{M d _ { 1 } \mathbf { v } _ { 2 }}\left(\mu \mathbf{K}_{\mu}+\nu \mathbf{K}_{\nu}\right) \mathbf{u}_{1}+3 \mathbf{n}\left(\mathbf{U}_{0}^{2} \mathbf{U}_{1}\right) \\
u_{3 A}=0, \quad u_{3 A}^{\prime}=0
\end{array}\right.
\end{aligned}
$$

The first perturbation equation (25 $)$ admits the (generating) not diverging solution

$\mathbf{U}_{0} \equiv\left\{\begin{array}{l}\mathbf{u}_{0} \\ \mathbf{v}_{0}\end{array}\right\}=a\left(t_{1}, t_{2}, \ldots\right)\left\{\begin{array}{c}\boldsymbol{\phi}_{1} \\ \mathbf{0}\end{array}\right\}$

where $\boldsymbol{\Phi}_{1}:=\left\{\boldsymbol{\phi}_{1}, \mathbf{0}\right\}^{\mathrm{T}}$ is an eigenvector for Eq. (6), and $a\left(t_{0}, t_{1}, \ldots\right)$ is a real unknown amplitude, which is modulated on the slower time scales. With Eqs. (26), the problem $\left(25_{2}\right)$ reads

$\varepsilon^{1 / 2}:\left\{\begin{array}{l}\mathrm{d}_{0} \mathbf{u}_{1} \quad \mathbf{v}_{1}=\mathrm{d}_{1} a \phi_{1} \\ \mathbf{M d}_{0} \mathbf{v}_{1}+\mathbf{K}_{0} \mathbf{u}_{1}+\mathbf{C} \mathbf{v}_{1}=\mathbf{0} \\ u_{1 A}=0, \quad u^{\prime}{ }_{1 A}=0\end{array}\right.$

Since the known term belongs to the range of the operator (recall Eqs. (13) and (27) admits the steady solution

$\mathbf{U}_{1} \equiv\left\{\begin{array}{l}\mathbf{u}_{1} \\ \mathbf{v}_{1}\end{array}\right\}=\mathrm{d}_{1} a\left(t_{1}, t_{2}, \ldots\right)\left\{\begin{array}{l}\phi_{2} \\ \phi_{1}\end{array}\right\}$

in which $\boldsymbol{\Phi}_{2}:=\left\{\boldsymbol{\phi}_{2}, \hat{\boldsymbol{\phi}}_{2}\right\}^{\mathrm{T}}=\left\{\boldsymbol{\phi}_{2}, \boldsymbol{\phi}_{1}\right\}^{\mathrm{T}}$ is the order 2 generalized eigenvector. With Eqs. (26) and (28), the problem (253) reads

$\varepsilon:\left\{\begin{array}{l}\mathrm{d}_{0} \mathbf{u}_{2} \mathbf{v}_{2}=\mathrm{d}_{2} a \phi_{1} \quad \mathrm{~d}_{1}^{2} a \boldsymbol{\phi}_{2} \\ \mathbf{M d}_{0} \mathbf{v}_{2}+\mathbf{K}_{0} \mathbf{u}_{2}+\mathbf{C} \mathbf{v}_{2}=\mathrm{d}_{1}^{2} a \mathbf{M} \phi_{1} a\left(\mu \mathbf{K}_{\mu}+\nu \mathbf{K}_{\nu}\right) \boldsymbol{\phi}_{1}+a^{3} \mathbf{n}\left(\boldsymbol{\Phi}_{1}^{3}\right) \\ u_{2 A}=0, \quad u_{2 A}^{\prime}=0\end{array}\right.$

In order it can be solved, the known term $\mathbf{F}_{2}:=\left\{-\mathrm{d}_{2} a \boldsymbol{\phi}_{1}-\mathrm{d}_{1}^{2} a \boldsymbol{\phi}_{2}\right.$, $\left.-\mathrm{d}_{1}^{2} a \mathbf{M} \boldsymbol{\phi}_{1}+a^{3} \mathbf{n}\left(\boldsymbol{\Phi}_{1}^{3}\right)-a\left(\mu \mathbf{K}_{\mu}+\nu \mathbf{K}_{\nu}\right) \boldsymbol{\phi}_{1}\right\}^{\mathrm{T}}$, must belong to the range of the operator; this solvability condition requires that

$\left\langle\boldsymbol{\Psi}, \mathbf{F}_{2}\right\rangle=0 \quad \forall \boldsymbol{\Psi}:\left(\mathbf{A}^{*} \bar{\lambda} \mathbf{B}^{*}\right) \boldsymbol{\Psi}=\mathbf{0}$

and it furnishes

$\mathrm{d}_{1}^{2} a=\left(c_{1 \mu} \mu+c_{1 \nu} \nu\right) a+c_{3} a^{3}$

where $c_{1 \mu}, c_{1 \nu}, c_{3}$ are real coefficients given in the Appendix B. By using Eq. (31) and solving Eq. (29), it follows:

$\mathbf{U}_{2} \equiv\left\{\begin{array}{l}\mathbf{u}_{2} \\ \mathbf{v}_{2}\end{array}\right\}=\mathrm{d}_{2} a\left\{\begin{array}{l}\phi_{2} \\ \phi_{1}\end{array}\right\}+\mu a\left\{\begin{array}{l}\mathbf{z}_{\mu} \\ \hat{\mathbf{z}}_{\mu}\end{array}\right\}+\nu a\left\{\begin{array}{l}\mathbf{z}_{\nu} \\ \hat{\mathbf{z}}_{\nu}\end{array}\right\}+a^{3}\left\{\begin{array}{l}\mathbf{z}_{a} \\ \hat{\mathbf{z}}_{a}\end{array}\right\}$

where $\mathbf{z}_{\mu}, \hat{\mathbf{z}}_{\mu}, \mathbf{z}_{\nu}, \hat{\mathbf{z}}_{\nu}, \mathbf{z}_{a}, \hat{\mathbf{z}}_{a}$ are solutions to linear problems (see Appendix B). To make the solution to the singular problem (29) unique, the normalization condition $u_{2 B}=0$ was enforced.

By using the results so far achieved, Eqs. $\left(25_{4}\right)$ read

$\varepsilon^{3 / 2}:\left\{\begin{array}{l}\mathrm{d}_{0} \mathbf{u}_{3} \quad \mathbf{v}_{3}=\mathrm{d}_{3} a \phi_{1} \quad \mathrm{~d}_{1} \mathrm{~d}_{2} a \phi_{2} \quad \mathrm{~d}_{1} \mathbf{u}_{2} \\ \mathbf{M d}_{0} \mathbf{v}_{3}+\mathbf{K}_{0} \mathbf{u}_{3}+\mathbf{C} \mathbf{v}_{3}=\mathrm{d}_{1} \mathrm{~d}_{2} a \mathbf{M} \boldsymbol{\phi}_{1} \\ \mathbf{M d}_{1} \mathbf{v}_{2} \quad \mathrm{~d}_{1} a\left(\mu \mathbf{K}_{\mu}+\nu \mathbf{K}_{\nu}\right) \phi_{2}+3 a^{2} \mathrm{~d}_{1} a \mathbf{n}\left(\boldsymbol{\Phi}_{1}^{2} \boldsymbol{\Phi}_{2}\right) \\ u_{3 A}=0, \quad u_{3 A}^{\prime}=0\end{array}\right.$

Its solvability condition entails

$2 \mathrm{~d}_{1} \mathrm{~d}_{2} a=\left(b_{1 \mu} \mu+b_{1 \nu} \nu\right) \mathrm{d}_{1} a+b_{3} a^{2} \mathrm{~d}_{1} a$

where $b_{1 \mu}, b_{1 \nu}, b_{3}$ are real coefficients (see Appendix B).

Finally, by coming back to the original time and quantities, Eqs. (31) and (34) are recombined, furnishing

$\ddot{a}\left[b_{1 \mu}\left(\begin{array}{ll}\mu & \mu_{0}\end{array}\right)+b_{1 \nu}\left(\nu \nu_{0}\right)\right] \dot{a} \quad b_{3} a^{2} \dot{a}$

$$
\left[c_{1 \mu}\left(\begin{array}{ll}
\mu & \mu_{0}
\end{array}\right)+c_{1 \nu}\left(\begin{array}{ll}
\nu & \nu_{0}
\end{array}\right)\right] a \quad c_{3} a^{3}=0
$$

which is the well known bifurcation equation for double zero bifurcation, in the Bogdanov normal form. In it, all the c's and $b$ 's coefficients depend on damping.

\subsection{Bifurcation scenarios}

The bifurcation equation (35) admits two equilibrium branches $a=a_{s}, \dot{a}_{s}=\ddot{a}_{s}=0, \forall t$ : (a) the trivial $a_{T}=0$, which exists in the 
whole $(\mu, \nu)$ plane and (b) the non trivial

$a_{N T}= \pm\left[\frac{c_{1 \mu}\left(\mu \mu_{0}\right) c_{1 \nu}\left(\nu \nu_{0}\right)}{c_{3}}\right]^{1 / 2}$

which exists in a half plane of the parameter space. Stability of both branches is governed by the variational equation

$\delta \ddot{a}+I_{1}\left(\mu, \nu ; a_{s}\right) \delta \dot{a}+I_{2}\left(\mu, \nu ; a_{s}\right) \delta a=0$

where

$I_{1}\left(\mu, \nu ; a_{s}\right):=\left[\begin{array}{ll}b_{1 \mu}\left(\begin{array}{ll}\mu & \mu_{0}\end{array}\right)+b_{1 \nu}\left(\begin{array}{ll}\nu & \nu_{0}\end{array}\right)+b_{3} a_{s}^{2}\end{array}\right]$

$\left.I_{2}\left(\mu, \nu ; a_{s}\right):=\left[\begin{array}{ll}c_{1 \mu}(\mu & \mu_{0}\end{array}\right)+c_{1 \nu}\left(\begin{array}{ll}\nu & \nu_{0}\end{array}\right)+3 c_{3} a_{s}^{2}\right]$

The trivial solution $a_{s}=a_{T}=0$ is considered first. It loses stability through divergence at the locus $\mathcal{D}:=\left\{(\mu, \nu) \mid I_{2}(\mu, \nu ; 0)=0\right\}$, and through Hopf bifurcation at the locus $\mathcal{H}_{T}:=\left\{(\mu, \nu) \mid I_{1}(\mu, \nu ; 0)=0\right.$, $\left.I_{2}(\mu, \nu ; 0)>0\right\}$, which are found to be a straight line and a half straight line, respectively, tangent to the exact $\mathcal{D}$ and $\mathcal{H}$ loci at the double zero point $D Z$.

When the non trivial solution $a_{s}=a_{N T}=0$ is considered, and use is made of Eq. (36), the divergence locus $I_{2}\left(\mu, \nu ; a_{N T}\right) \equiv$ $-2 I_{2}(\mu, \nu ; 0) \equiv-2 c_{3} a_{N T}^{2}=0$ turns out to be coincident with the line $\mathcal{D}$ previously defined, this entailing that the non trivial solution does not undergo any other divergence, in addition to that one from which it arises. Moreover, since the stable zone lies in the half plane $I_{2}(\mu, \nu ; 0)>0$, the static bifurcation is supercritical if $c_{3}<0$, and subcritical if $c_{3}>0$. In contrast, the non trivial solution experiences a Hopf bifurcation at the half straight line $\mathcal{H}_{N T}:=$ $\left\{(\mu, \nu) \mid I_{1}\left(\mu, \nu ; a_{N T}\right)=0, I_{2}\left(\mu, \nu ; a_{N T}\right)>0\right\}$. The inequality entails the existence condition $c_{3}<0$; if this is the case, $\mathcal{H}_{T}$ and $\mathcal{H}_{N T}$ lie in the half planes $I_{2}(\mu, \nu ; 0)>0$ and $I_{2}(\mu, \nu ; 0)<0$, respectively, which are separated by the line $\mathcal{D}$.

\subsection{Parametric analysis}

The previous qualitative analysis highlights the strong depen dence of the bifurcation scenario on the sign of the coefficient $c_{3}$. In order to investigate the influence of damping, this coefficient has been evaluated for different values of $\alpha$ and $\beta$, and results displayed in Table 1 . It is seen that, $c_{3}<0$ when $\beta$ is fixed at a small value (weakly externally damped systems) and $\alpha$ is varied on a range; in contrast, the coefficient changes sign when $\beta$ is suffi ciently large (strongly externally damped systems). A similar analysis carried out on the coefficient $b_{3}$ shows that this coefficient is always negative, this entailing that the Hopf bifurcation is always supercritical. Moreover, Fig. 4 shows a qualitative repre sentation of the bifurcation chart for varying $\beta$ coefficient causing a change in the sign of $c_{3}$ coefficient. It should be highlighted the fact that homoclinic and heteroclinic bifurcations occur, as it will be shown ahead, and that in the transition, Divergence, non trivial Hopf and homoclinic Hopf tend to overlap themselves, this giving rise to the birth of heteroclinic Hopf and to change radically the region of existence of non trivial equilibrium (denoted in gray in the figure).

Guided by these results, four sample system have been con sidered, in which the internal damping parameter has been fixed,

Table 1

Coefficient $c_{3}$ in the bifurcation equation vs. damping coefficients $\alpha$ and $\beta$.

\begin{tabular}{|c|c|c|c|c|}
\hline $\begin{array}{lr}\alpha & 0.01 \\
-29.707\end{array}$ & $\begin{array}{lr}\alpha & 0.05 \\
-35.669\end{array}$ & $\begin{array}{cc}\beta & 0.1 \\
\alpha & 0.1 \\
-30.692\end{array}$ & $\begin{array}{lr}\alpha & 0.8 \\
-2.348\end{array}$ & $\begin{array}{lr}\alpha & 1.5 \\
-0.703\end{array}$ \\
\hline $\begin{array}{ll}\beta & 0.25 \\
-3.221\end{array}$ & 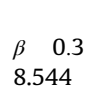 & $\begin{array}{lc}\alpha & 0.01 \\
\beta & 0.5 \\
62.603\end{array}$ & $\begin{array}{l}\beta \quad 1 \\
235.510\end{array}$ & $\begin{array}{l}\beta \quad 10 \\
574.504\end{array}$ \\
\hline
\end{tabular}
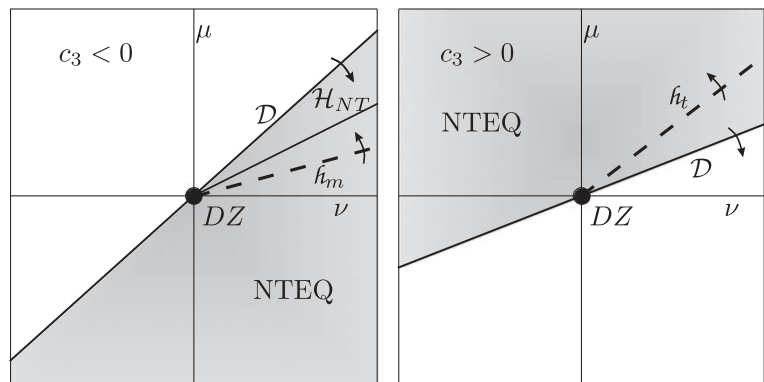

Fig. 4. Qualitative representation of the bifurcation chart in the parameter plane and of loci transition in varying external damping for: (a) $c_{3}<0$; (b) $c_{3}>0 ; h_{m}$ homoclinic bifurcation locus; $h_{t}$ heteroclinic bifurcation; $\mathcal{D}$ divergence locus; $\mathcal{H}_{N T}$ non-trivial Hopf bifurcation locus; NTEQ region of existence of non-trivial equilibrium.

while the external one has been varied in a wide range. As it will shown ahead the first two systems exhibit supercritical static bifurcation, and in particular, in the second one the parameter set is very close to the change in sign of the $c_{3}$ coefficient (but it is still $c_{3}<0$ ); moreover, the third and the fourth systems exhibit subcritical static bifurcation and again the parameter set of the third system is close to the transition zone for the sign of the $c_{3}$ coefficient (but, in this case $c_{3}>0$ ). Finally, the first and the fourth systems coincide with those presented in [39]. The para meter sets are

(S1) for which $\alpha=0.01, \beta=0.1$, entailing $\mu_{0}=2.806, \nu_{0}=2.884$ and $c_{1 \mu}=-10.641, \quad c_{1 \nu}=6.907, \quad c_{3}=-29.707, \quad b_{1 \mu}=0.246$, $b_{1 \nu}=0.127, b_{3}=-6.077$.

(S2) for which $\alpha=0.01, \beta=0.25$, entailing $\mu_{0}=3.022, \nu_{0}=3.302$ and $c_{1 \mu}=-14.241, \quad c_{1 \nu}=5.605, \quad c_{3}=-3.221, \quad b_{1 \mu}=0.445$, $b_{1 \nu}=0.251, b_{3}=-15.154$.

(S3) for which $\alpha=0.01, \beta=0.3$, entailing $\mu_{0}=3.063, \nu_{0}=3.416$ and $\quad c_{1 \mu}=-15.449, \quad c_{1 \nu}=5.189, \quad c_{3}=8.544, \quad b_{1 \mu}=0.526$, $b_{1 \nu}=0.301, b_{3}=-19.451$.

(S4) for which $\alpha=0.01, \beta=10$, entailing $\mu_{0}=3.076, \nu_{0}=5.302$ and $c_{1 \mu}=-22.324, c_{1 \nu}=-5.156, c_{3}=574.504, b_{1 \mu}=4.522$, $b_{1 \nu}=6.014, b_{3}=-1153.303$.

Results relevant to S1 are reported in Fig. 5a, displaying the bifurcation chart, and Fig. 6, illustrating bifurcation diagrams, these latter having been obtained by a numerical continuation procedure. Fig. 5a shows also the tangency between asymptotic and exact bifurcation loci (gray curves); here, in each significant region, the two dimensional phase plane $(a, \dot{a})$ for the bifurcation equation (35) is sketched. In region 1 the trivial solution is stable; due to the supercritical static bifurcation, it loses stability in region 2, where two (buckled) stable non trivial equilibria take place; due to supercritical Hopf bifurcation, it loses stability in region 5, where a (large) stable limit cycle exists, causing periodic motion of the beam. In region 4 two equilibria appear, but, in spite of the supercritical character of the static bifurcation, they are unstable, as an effect of the interaction with the dynamic bifurcation; in region 3 two small unstable limit cycles arise (denoting periodic motions around the buckled configurations), which render stable the non trivial equilibria. Then, at the straight line $h_{m}$, a homo clinic bifurcation occurs (caused by the contact of the small cycles with the trivial equilibrium and, simultaneously, with the large cycles); after that, all cycles disappear, so that only stable equili bria survive in region 2. Fig. 6 shows the bifurcation diagrams relevant to the paths I to IV marked in Fig. 5a; labels $D, H, h_{m}$ denote divergence, Hopf and homoclinic bifurcation, respectively. Path I shows the static bifurcation, from which stable non trivial 

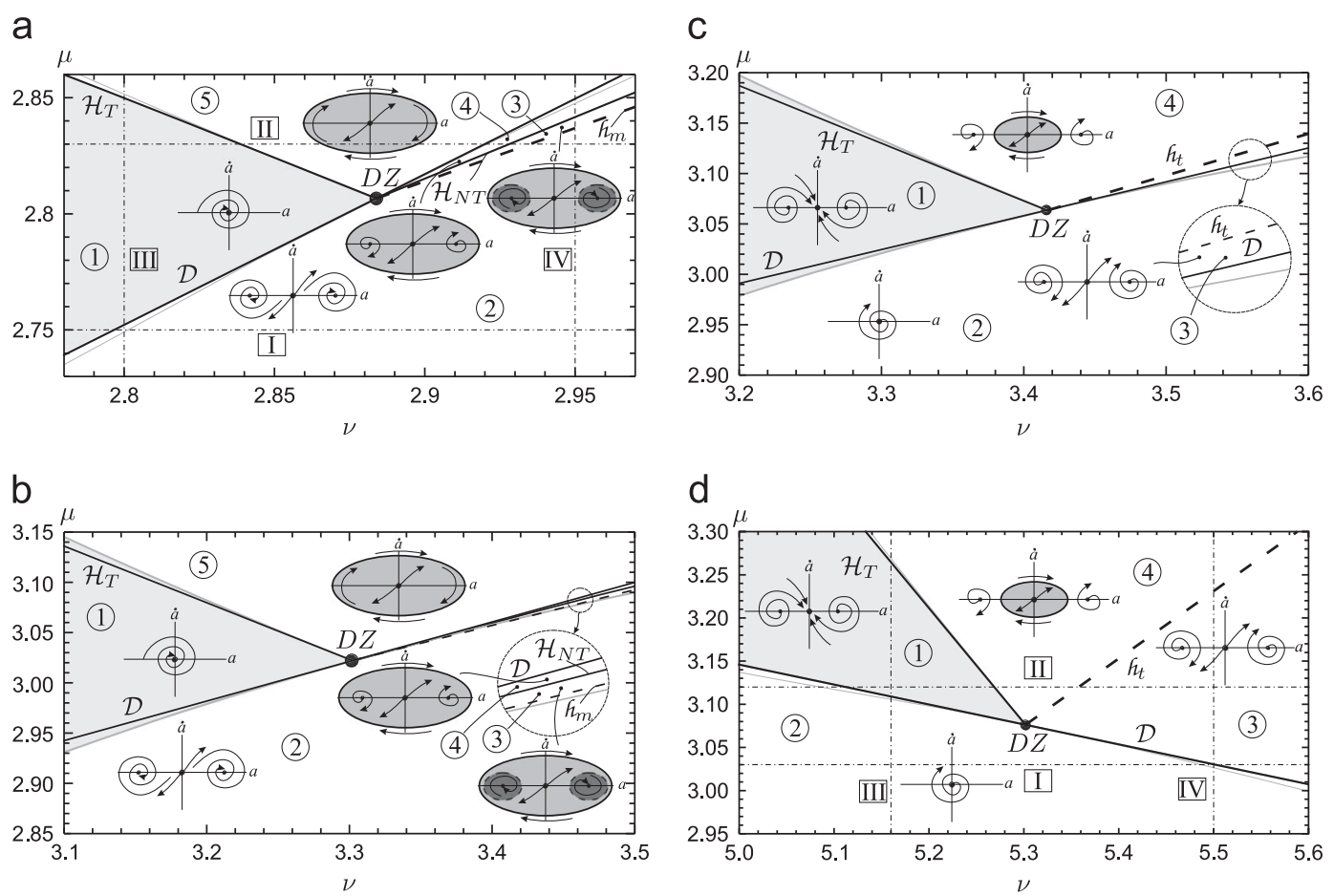

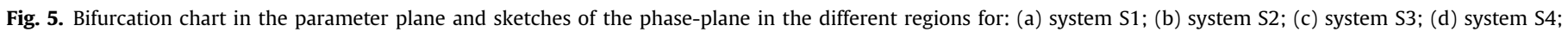
$h_{m}$ homoclinic bifurcation locus; $h_{t}$ heteroclinic bifurcation locus.
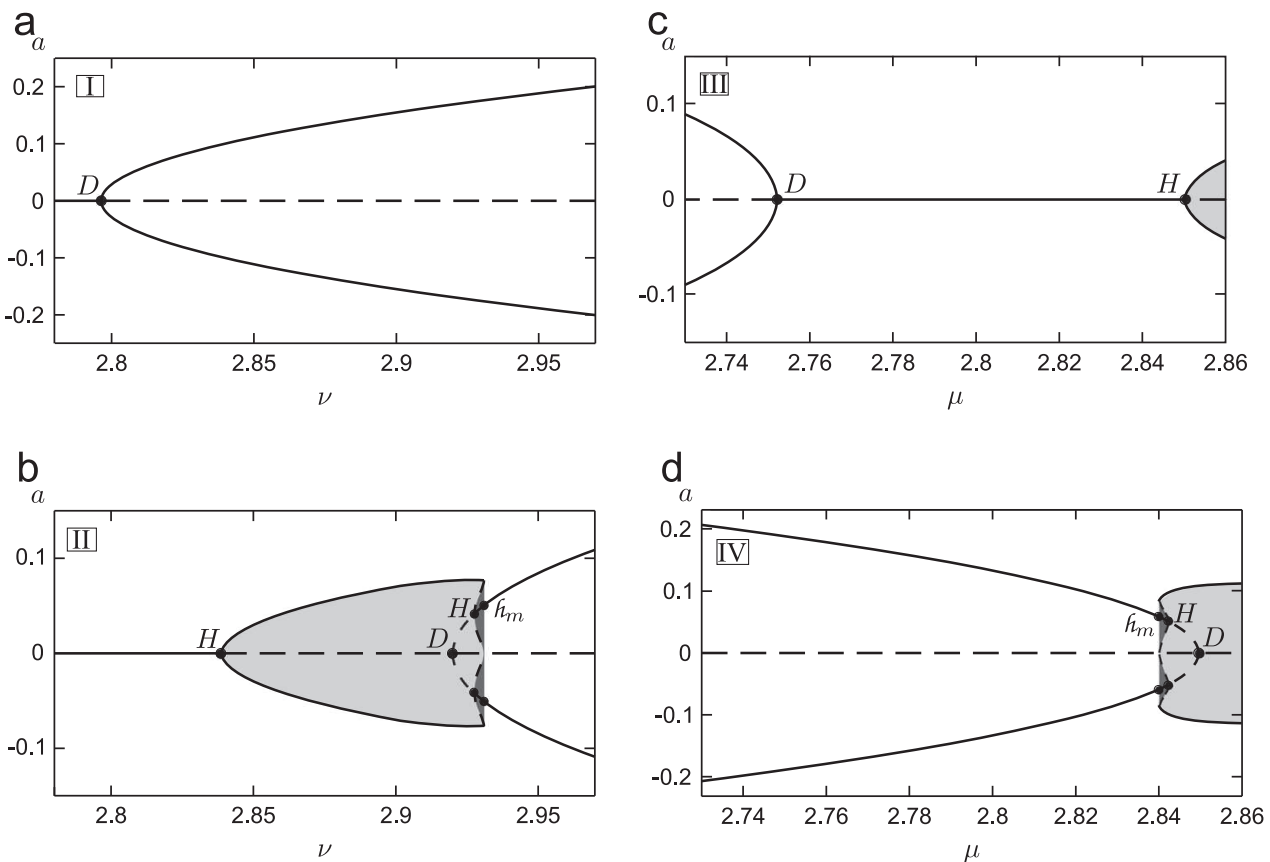

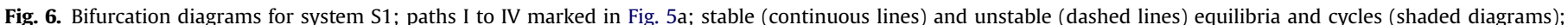
labels $D, H, h_{m}$ denote divergence, Hopf and homoclinic bifurcation points, respectively.

equilibria arise. Path II displays, in sequence, (a) the Hopf bifurca tion from the trivial solution, leading to the appearance of large cycles, whose amplitude range is shadow in the figure; (b) the static bifurcation, leading to initially unstable non trivial equili bria; (c) the Hopf bifurcation from the non trivial equilibria, leading to the appearance of small cycles and the simultaneous regain of stability of the non trivial equilibria; (d) the homoclinic bifurcation, causing the disappearing of all the cycles. Path III illustrates the loss of stability of the trivial equilibrium through divergence (for small $\mu$ ) or Hopf bifurcation (for large $\mu$ ). Finally, path IV shows the transition from non trivial equilibria to large limit cycles, passing through homoclinic bifurcation. As a final comment on the scenario relevant to S1, there exist an attractor in any region, namely: one or two equilibria in regions 1 and 2, one cycle in regions 4 and 5, and two equilibria and a cycle in region 3 . Therefore the bifurcation is not catastrophic. On the other hand, the regions ( 3 and 4 ) in which static and dynamic bifurcations interact are of small extension. 

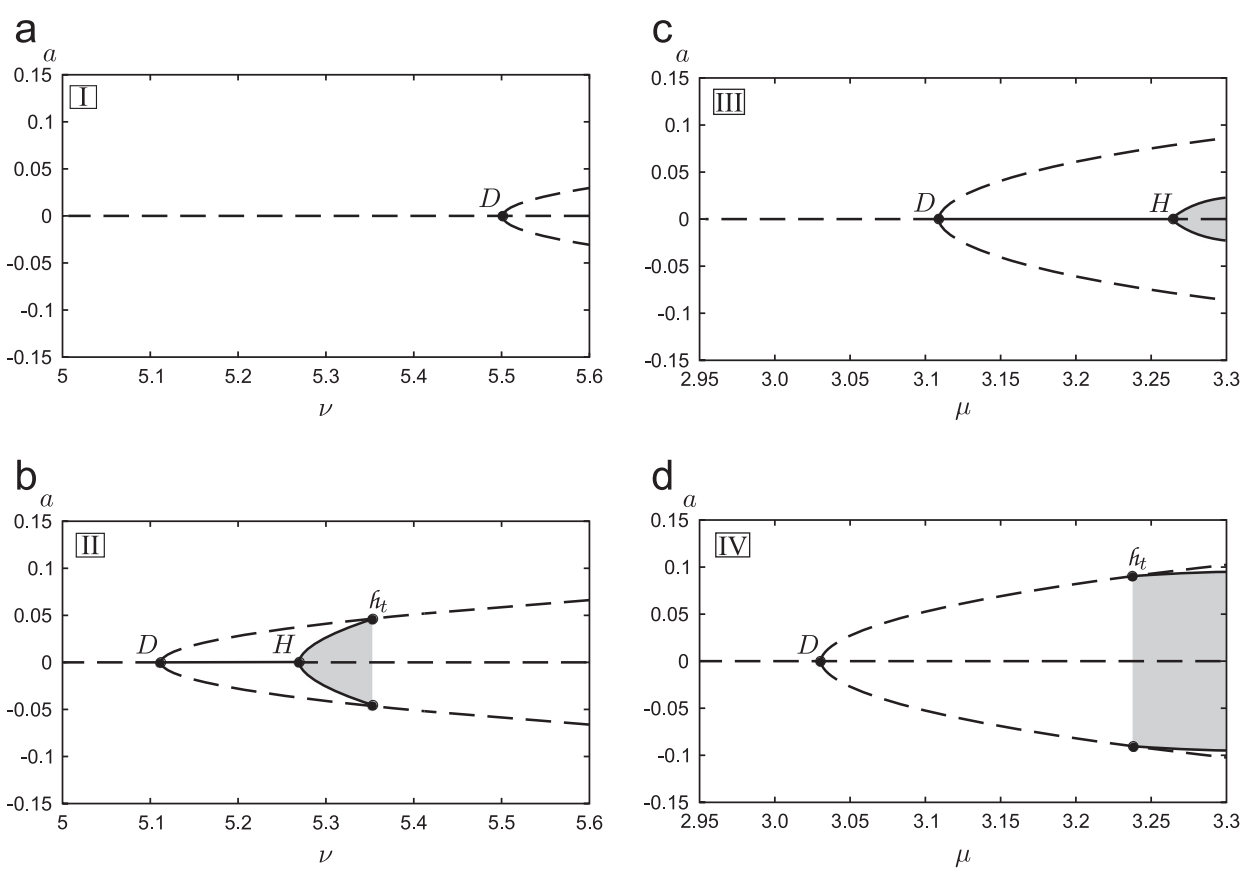

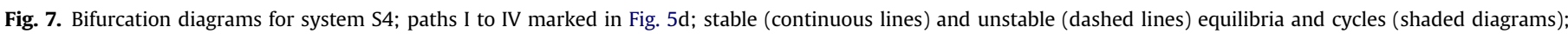
labels $D, H, h_{t}$ denote divergence, Hopf and heteroclinic bifurcation points, respectively.

System S2, in Fig. 5b exhibits the same qualitative behavior of S1 but the areas of regions 3 and 4 appear significantly reduced, due to the fact that coefficient $c_{3}$ is closer to zero with respect to what happens in S1. However, also in this case, both the two unstable equilibria (region 4 ) both the two small unstable limit cycles, which again render stable the non trivial equilibria (in region 3 ), survive and a homoclinic bifurcation, denoted by the line $h_{m}$, again manifests itself at the contact of the small cycles with the trivial equilibrium.

Since system S3, in Fig. $5 \mathrm{c}$ exhibits the same qualitative behavior of $\mathrm{S} 4$, but it is close to the transition zone of the $c_{3}$ coefficient, here system S4 is first described. Numerical results relevant to system S4 are reported in Figs. $5 d$ and 7. Since $c_{3}>0$, the static bifurcation is subcritical and, according to the previous qualitative analysis, the bifurcated equilibria do not suffer Hopf bifurcation, so that no a curve $\mathcal{H}_{N T}$ exists and, consequently, no homoclinic bifurcation $h_{m}$ occurs. In contrast, a new heteroclinic bifurcation $h_{t}$ manifests itself. In region 1 (Fig. 5d) the trivial equilibrium is stable, but two unstable equilibrium points coexist. In region 2 the trivial equilibrium loses stability by divergence, and no other local attractors exist. In region 4 the equilibrium loses stability by supercritical Hopf bifurcation, giving rise to a stable limit cycle internal to the non trivial equilibria. In region 3 , however, due to a heteroclinic bifurcation caused by the collision of cycle with the non trivial equilibria, the cycle itself disappears. Paths I and IV (Fig. 7) show the static bifurcation; path II illustrates the succession of (a) static, (b) Hopf and (c) heteroclinic bifurca tions; path III illustrates the loss of stability by divergence (small $\mu$ ) or Hopf bifurcation (large $\mu$ ). Therefore, system S2 has stable attractors only in region 1 (trivial equilibrium) and 4 (limit cycle); in the remaining regions no attractors exist. The bifurcation is therefore catastrophic.

By coming back to system S3, in Fig. $5 c$, since $c_{3}>0$ but lower with respect to what happen in system S4, region 3 appears reduced while region 4 is enlarged. However, also in this case, the equilibrium loses stability by supercritical Hopf bifurcation and a stable limit cycle, internal to the non trivial equilibria, exists (region 4), but, due to the heteroclinic bifurcation, denoted again by the line $h_{t}$, the cycle itself disappears (region 3 ).

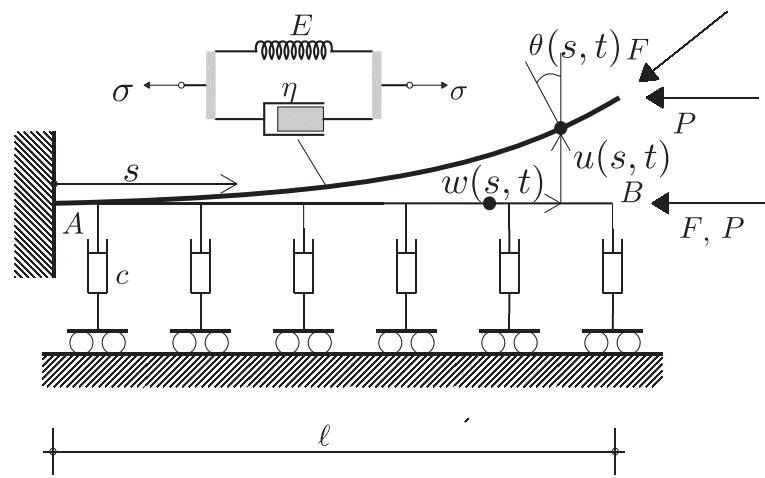

Fig. 8. Visco-elastic beam on viscous soil under follower force and dead load: model and displacements.

\section{Conclusions}

A non linear, visco elastic, externally damped column, subjected to two independent axial loads, one gravitational and the other tangential, has been studied. By enforcing internal kinematical constrains, a single non linear integro differential equation of motion in the transversal displacement field has been derived, equipped with proper boundary conditions. The linear stability diagram of the trivial equilibrium has been studied in detail in the plane of the two loading parameters, both for tensile and compressive forces. The existence of divergence and Hopf bifurcations has been highlighted, leading to double zero (Takens Bogdanov) bifurcations. A non linear bifurcation analysis, based on a fractional power version of the multiple scale method, has been performed around the double zero point, by directly attacking the continuous problem (i.e. by avoiding any a priori discretization). A parametric analysis has been carried out, with the aim to investigate the role of damping on such a codimension 2 bifurcation, both in linear and non linear problems. The following main conclusions are drawn:

1. The position of the bifurcation point and the angle of attack between the incident, divergence and Hopf, bifurcation loci 
depend on the damping coefficients. However, when one of the coefficients is zeroed and the other is rendered small, the properties of the undamped (circulatory) system are recovered only for evanescent external damping, not for internal damp ing, this case being in discontinuity with the circulatory case. Therefore, some new features of the well known "destabiliza tion paradox" are revealed.

2. The non linear scenario around the double zero bifurcation is strongly affected by damping. When the external damping is small, the static bifurcation is supercritical, this entailing the existence of one or more attractor, equilibria or limit cycles, in the whole neighborhood. In contrast, when the external damping is large, the static bifurcation is subcritical, this entailing a catastrophic character of the bifurcation, for the lack of attractors in some region around the bifurcation point. In the whole range studied, instead, the Hopf bifurcation has supercritical character.

3. The interaction between static and dynamic bifurcations man ifests itself via homoclinic or heteroclinic bifurcations, due to the collision between limit cycles and equilibria, or between cycles.

\section{Acknowledgments}

This work was supported by the Italian Ministry of University (MIUR) through the PRIN cofinanced program no. 2010MBJK5B.

\section{Appendix A}

In this Appendix A derivation of the equation of motion for the structural model, by using the extended Hamilton's principle, is presented.

A planar beam is considered, fixed at the end $A$, and simulta neously loaded at the tip $B$ by a follower force of intensity $F$ (tangential to the actual configuration of the beam axis) and by a dead load of intensity $P$ (acting in the direction of the originally rectilinear axis, Fig. 8). The material behavior of the beam obeys to the Kelvin Voigt rheological model, with elastic modulus $E$ and viscous coefficient $\eta$ (acting as an internal damping); moreover, the beam is considered to lie on a purely viscous linear soil of constant $c$ (simulating the external damping). The beam is assumed to be inextensible and shear undeformable, Formulation closely follows the procedure of [41], where a similar system was considered, with, however, no dead load nor internal damping, but a lumped visco elastic device.

The actual configuration of the beam is described by the transversal displacement field $u(s, t)$, the longitudinal displace ment $w(s, t)$ of the beam axis, and moreover, the rotation of the section $\vartheta(s, t)$, where $s \in[0, \ell]$ is a curvilinear abscissa and $t$ is the time. The three displacements, however, are not independent, because of the internal constraints, expressing no shear and no stretch, respectively

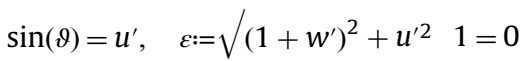

where a dash denotes differentiation with respect to $s$. The curvature $\kappa(s, t)$ is assumed as the (unique) strain measure; from Eq. (A. $1_{1}$ ) it follows that:

$\kappa:=\vartheta^{\prime}=\frac{u^{\prime \prime}}{\sqrt{ } 1 u^{\prime 2}}$

The equations of motion are derived by the extended Hamilton's principle, by introducing the constraint equation (A.1 $1_{2}$ ) by a Lagrangian multiplier $R(s, t)$, having the meaning of (reactive) horizontal force. The variational principle reads

$$
\begin{aligned}
\delta H= & \int_{t_{1}}^{t_{2}} \int_{0}^{\ell}[m(\dot{u} \delta \dot{u}+\dot{w} \delta \dot{w}) E I \kappa \delta \kappa \quad \eta I \dot{\kappa} \delta \kappa \\
& c \dot{u} \delta u \quad \delta(R \varepsilon)] d s d t+\int_{t_{1}}^{t_{2}}\left[\left(P+F \cos \left(\vartheta_{B}\right)\right) \delta w_{B}\right. \\
& \left.F \sin \left(\vartheta_{B}\right) \delta u_{B}\right] d t=0, \quad \forall(\delta u, \delta w, \delta R)
\end{aligned}
$$

where $E I$ is the flexural stiffness of the beam, a dot denotes time differentiation, and the index $B$ evaluation at $s=\ell$. By using Eq. (A. $1_{1}$ ) to eliminate the rotation $\vartheta(s)$, expanding $u(s)$ in Taylor series, the equations of motion, corrected up to the third order, are derived. By introducing the following non dimensional quantities:

$\tilde{t}=\omega t, \quad \tilde{s}=s / \ell, \quad \tilde{u}=u / \ell, \quad \tilde{w}=w / \ell$

$\tilde{R}=R / m \ell^{2} \omega^{2}, \quad \omega^{2}=E I / m \ell^{4}, \quad \alpha=\eta \omega / E$

$\beta=c \omega \ell^{4} / E I, \quad \mu=F \ell^{2} / 2 E I, \quad \nu=P \ell^{2} / 2 E I$

omitting the tilde symbol, and still denoting by dashes and dots differentiations with respect to non dimensional quantities, the equations read

$\ddot{u}+u^{\mathrm{IV}}+\left[u^{\prime}\left(u^{\prime} u^{\prime \prime}\right)^{\prime}\right]^{\prime}+\alpha \dot{u}^{\mathrm{IV}}+\alpha\left\{\left[u^{\prime}\left(u^{\prime} u^{\prime \prime}\right)^{\prime}\right]^{\prime}\right\}^{\bullet}+\beta \dot{u} \quad\left(R u^{\prime}\right)^{\prime}=0$

$\ddot{w} \quad R^{\prime}=0$

$w^{\prime}+\frac{u^{\prime 2}}{2}=0$

with the relevant boundary conditions, of geometrical type

$w_{A}=0, \quad u_{A}=0, \quad u_{A}^{\prime}+\frac{1}{6} u_{A}^{\prime 3}=0$

and mechanical type

$$
\begin{gathered}
R_{B}+2 \mu\left(1 \frac{u_{B}^{\prime 2}}{2}\right)+2 \nu=0 \\
{\left[\left(u_{B}^{\prime \prime \prime}+u_{B}^{\prime \prime} u_{B}^{\prime 2}+u^{\prime \prime 2}{ }_{B}^{\prime} u_{B}^{\prime}\right)\left(2 \mu+R_{B}\right) u_{B}^{\prime}\right.} \\
\left.+\alpha\left(u_{B}^{\prime \prime}+u_{B}^{\prime \prime} u_{B}^{\prime 2}+u^{\prime \prime 2}{ }_{B}^{\prime} u_{B}\right)^{\bullet}\right]=0 \\
\left(u_{B}^{\prime \prime}+u_{B}^{\prime \prime} u_{B}^{\prime 2}\right)+\alpha\left(u_{B}^{\prime \prime}+u_{B}^{\prime \prime} u_{B}^{\prime 2}\right)^{\bullet}=0
\end{gathered}
$$

In deriving Eqs. (A.5) (A.7), $R^{\prime}$ was considered as a second order quantity, in accordance with Eqs. (A.5 2,3 ), so that terms as $R^{\prime} u^{\prime 2}$ were neglected.

The longitudinal displacement $w(s, t)$ and the force $R(s, t)$ follow from integration of Eqs. (A.5 $5_{2,3}$ ) with the boundary conditions $\left(\right.$ A. $\left.6_{1}\right)$ and $\left(A .7_{1}\right)$, namely

$w=\frac{1}{2} \int_{0}^{s} u^{\prime 2} d s$

$R=2 \mu\left(1 \frac{u_{B}^{\prime 2}}{2}\right) 2 \nu \frac{1}{2} \int_{1}^{s}\left(\int_{0}^{s} u^{\prime 2} d s\right)^{\bullet \bullet} d s$

By substituting Eq. (A.8 $)$ in Eq. (A.5 $)$ and in the remaining boundary conditions, the following (condensed) equations in the unique variable $u(s, t)$ are finally derived

$$
\begin{aligned}
& \ddot{u}+u^{I V}+\left[u^{\prime}\left(u^{\prime} u^{\prime \prime}\right)^{\prime}\right]^{\prime} \\
& +\dot{\alpha} u^{I V}+\alpha\left\{\left[u^{\prime}\left(u^{\prime} u^{\prime \prime}\right)^{\prime}\right]^{\prime}\right\} \\
& +2\left[\mu\left(1 \frac{u_{B}^{\prime 2}}{2}\right)+v\right] u^{\prime \prime}+\beta \dot{u}+ \\
& \frac{1}{2}\left[\left(\int_{1}^{s}\left(\int_{0}^{s} u^{\prime 2} d s\right)^{\bullet \bullet} d s\right) u^{\prime}\right]^{\prime}=0 \\
& u_{A}=0, \quad u_{A}^{\prime}+\frac{1}{6} u_{A}^{3}=0 \\
& {\left[\left(u_{B}^{\prime \prime \prime}+u_{B}^{\prime \prime \prime} u_{B}^{\prime 2}+u_{B}^{\prime \prime 2} u_{B}^{\prime}\right)+2\left(v \mu \frac{u_{B}^{\prime 2}}{2}\right) u_{B}^{\prime}\right.} \\
& \left.+\alpha\left(u_{B}^{\prime \prime \prime}+u_{B}^{\prime \prime \prime} u_{B}^{\prime 2}+u_{B}^{\prime \prime 2} u_{B}^{\prime}\right)^{\bullet}\right]=0 \\
& \left(u_{B}^{\prime \prime}+u_{B}^{\prime \prime} u_{B}^{\prime 2}\right)+\alpha\left(u_{B}^{\prime \prime}+u_{B}^{\prime \prime} u_{B}^{\prime 2}\right)^{\bullet}=0
\end{aligned}
$$


The obtained equations (A.9) are the same of those presented in [39]; they are of integro differential type and contain cubic non linearities only. It should be noticed that linearization of the geometrical conditions does not entail any error at this order.

Finally, Eqs. (A.9) can be recasted in an operator form, in which the mechanical boundary conditions are appended to the field equations: in this way Eqs. (1) are obtained.

\section{Appendix B}

The $\mathbf{z}$ solutions appearing in Eq. (32) satisfy the following linear problems:

$$
\begin{aligned}
& \left\{\begin{array}{l}
\mathrm{d}_{0} \mathbf{z}_{\mu} \hat{\mathbf{z}}_{\mu}=c_{1 \mu} \boldsymbol{\phi}_{2} \\
\mathbf{M d}_{0} \hat{\mathbf{z}}_{\mu}+\mathbf{K}_{0} \mathbf{z}_{\mu}+\mathbf{C} \hat{\mathbf{z}}_{\mu}=c_{1 \mu} \mathbf{M} \boldsymbol{\phi}_{1} \quad \mathbf{K}_{\mu} \boldsymbol{\phi}_{1} \\
z_{\mu A}=0, \quad z_{\mu A}^{\prime}=0
\end{array}\right. \\
& \left\{\begin{array}{l}
\mathrm{d}_{0} \mathbf{z}_{\nu} \hat{\mathbf{z}}_{\nu}=c_{1 \nu} \boldsymbol{\phi}_{2} \\
\mathbf{M d}_{0} \hat{\mathbf{z}}_{\nu}+\mathbf{K}_{0} \mathbf{z}_{\nu}+\mathbf{C} \hat{\mathbf{z}}_{\nu}=c_{1 \nu} \mathbf{M} \boldsymbol{\phi}_{1} \quad \mathbf{K}_{\nu} \boldsymbol{\phi}_{1} \\
z_{\nu A}=0, \quad z_{\nu A}^{\prime}=0
\end{array}\right. \\
& \left\{\begin{array}{l}
\mathrm{d}_{0} \mathbf{z}_{a} \hat{\mathbf{z}}_{a}=c_{3} \boldsymbol{\phi}_{2} \\
\mathbf{M d}_{0} \hat{\mathbf{z}}_{a}+\mathbf{K}_{0} \mathbf{z}_{a}+\mathbf{C} \hat{\mathbf{z}}_{a}=c_{3} \mathbf{M} \boldsymbol{\phi}_{1}+\mathbf{n}\left(\boldsymbol{\Phi}_{1}^{3}\right) \\
z_{a A}=0, \quad z_{a A}^{\prime}=0
\end{array}\right.
\end{aligned}
$$

under the normalization conditions

$z_{\mu B}=0, \quad z_{\nu B}=0, \quad z_{a B}=0$

Due to their cumbersome expressions, they are not reported here. Coefficients in Eq. (35) take the following forms:

$c_{1 \mu}=2 \int_{0}^{1} \psi_{2} \phi^{\prime \prime}{ }_{1} \mathrm{~d} s$

$c_{1 \nu}=2 \int_{0}^{1} \psi_{2} \phi^{\prime \prime}{ }_{1} \mathrm{~d} s+2 \psi_{2 B} \phi^{\prime}{ }_{1 B}$

$c_{3}=\int_{0}^{1} \psi_{2} n_{1}\left(\boldsymbol{\Phi}_{1}^{3}\right) \mathrm{d} s+\psi_{2 B} n_{2}\left(\boldsymbol{\Phi}_{1}^{3}\right)+\psi^{\prime}{ }_{2 B} n_{3}\left(\boldsymbol{\Phi}_{1}^{3}\right)$

and

$$
\begin{aligned}
b_{1 \mu}= & \int_{0}^{1}\left[\left(\alpha \psi_{2}^{\mathrm{IV}}+\beta \psi_{2}\right) z_{\mu}+\psi_{2} \hat{z}_{\mu}+2 \psi_{2} \phi^{\prime \prime}{ }_{2}\right] \mathrm{d} s+\alpha \psi^{\prime \prime \prime}{ }_{2 B} z_{\mu B} \quad \alpha \psi^{\prime \prime}{ }_{2 B} z_{\mu B}^{\prime} \\
b_{1 \nu}= & \int_{0}^{1}\left[\left(\alpha \psi_{2}^{\mathrm{IV}}+\beta \psi_{2}\right) z_{\nu}+\psi_{2} \hat{z}_{\nu}+2 \psi_{2} \phi^{\prime \prime}{ }_{2}\right] \mathrm{d} s \\
& +\alpha \psi^{\prime \prime \prime}{ }_{2 B} z_{\nu B} \quad \alpha \psi^{\prime \prime}{ }_{2 B} z^{\prime}{ }_{\nu B}+2 \psi_{2 B} \phi^{\prime}{ }_{2 B} \\
b_{3}= & 3 \int_{0}^{1}\left(\alpha \psi_{2}^{\mathrm{IV}}+\beta \psi_{2}\right) z_{a}+\psi_{2} \hat{z}_{a} \\
& \psi_{2} n_{1}\left(\boldsymbol{\Phi}_{1}^{2} \boldsymbol{\Phi}_{2}\right) \mathrm{d} s+3\left[\alpha \psi^{\prime \prime \prime}{ }_{2 B} z_{a B}\right. \\
& \left.\alpha \psi^{\prime \prime}{ }_{2 B} z_{a B}^{\prime}+\psi_{2 B} n_{2}\left(\boldsymbol{\Phi}_{1}^{2} \boldsymbol{\Phi}_{2}\right)+\psi_{2 B}^{\prime} n_{3}\left(\boldsymbol{\Phi}_{1}^{2} \boldsymbol{\Phi}_{2}\right)\right]
\end{aligned}
$$

\section{References}

[1] M. Beck, Die knicklast des einseiting eingespannten tangential gedrckten stabes, Zeitschrift für Angewandte Mathematik und Physik 3 (1952) 225-228.

[2] V.V. Bolotin, Nonconservative Problems of the Theory of Elastic Stability, Macmillan, New York, 1963.

[3] B.R. Ryu, K. Katayama, Y. Sugiyama, Dynamic stability of timoshenko columns subjected to subtangential forces, Computers \& Structures 68 (1998) 499-512.

[4] M.A. Langthjem, Y. Sugiyama, Dynamic stability of columns subjected to follower loads: a survey, Journal of Sound and Vibration 238 (2000) 809-851.

[5] A. Mazidi, S.A. Fazelzadeh, P. Marzocca, Flutter of aircraft wings carrying a powered engine under roll maneuver, Journal of Aircraft 48 (2011) 874-883.

[6] S.A. Fazelzadeh, A. Mazidi, Nonlinear aeroelastic analysis of bending-torsion wings subjected to a transverse follower force, Journal of Computational and Nonlinear Dynamics, http://dx.doi.org/10.1115/1.4003288, in press.

[7] Q. Wang, S.T. Quek, Enhancing flutter and buckling capacity of column by piezoelectric layers, International Journal of Solids and Structures 39 (2002) 4167-4180.

[8] Y. Wang, W. Zhongmin, Z. Lei, Stability of viscoelastic rectangular plate with a piezoelectric layer subjected to follower force, Archive of Applied Mechanics 1 (2012) 1-13.
[9] O. Kayacik, J.C. Bruch Jr., J.M. Sloss, S. Adali, I. Sadek, Integral equation approach for piezo patch vibration control of beams with various types of damping, Computers \& Structures 86 (2008) 357-366.

[10] S.A. Fazelzadeh, M. Eghtesad, M. Azadi, Buckling and flutter of a column enhanced by piezoelectric layers and lumped mass under a follower force, International Journal of Structural Stability and Dynamics 10 (2010) 1083-1097.

[11] U. Andreaus, F. dell'Isola, M. Porfiri, Piezoelectric passive distributed controllers for beam flexural vibrations, Journal of Vibration and Control 10 (2004) 625-659.

[12] C. Maurini, F. dell'Isola, D. Del Vescovo, Comparison of piezoelectronic networks acting as distributed vibration absorbers, Mechanical Systems and Signal Processing 18 (2004) 1243-1271.

[13] F. dell'Isola, S. Vidoli, Continuum modelling of piezoelectromechanical truss beams: an application to vibration damping, Archive of Applied Mechanics 68 (1998) 1-19.

[14] F. dell'Isola, S. Vidoli, Damping of bending waves in truss beams by electrical transmission lines with pzt actuators, Archive of Applied Mechanics 68 (1998) 626-636.

[15] L. Wang, Flutter instability of supported piped conveying fluid subjected to distributed follower forces, Acta Mechanica Sinica 25 (2012) 46-52.

[16] S.A. Fazelzadeh, M.A. Kazemi-Lari, Stability analysis of partially loaded leipholz column carrying a lumped mass and resting on elastic foundation, Journal of Sound and Vibration 332 (2013) 595-607.

[17] J.T. Katsikadelis, G.C. Tsiatas, Non-linear dynamic stability of damped beck's column with variable cross-section, International Journal of Nonlinear Mechanics 42 (2007) 164-171.

[18] G.C. Tsiatas, J.T. Katsikadelis, Post-critical behavior of damped beam columns with variable cross section subjected to distributed follower forces, NonLinear Dynamics 56 (2008) 429-441.

[19] D. Sophianopoulos, A. Kounadis, A. Vakakis, Complex dynamics of perfect discrete systems under partial follower forces, International Journal of Nonlinear Mechanics 37 (7) (2002) 1121-1138.

[20] F. dell'Isola, K. Hutter, A qualitative analysis of the dynamics of a sheared and pressurized layer of saturated soil, Proceedings of the Royal Society of London A Mathematical 454 (1998) 3105-3120.

[21] F. dell'Isola, A. Madeo, P. Seppecher, Boundary conditions at fluid-permeable interfaces in porous media: a variational approach, International Journal of Solids and Structures 46 (2009) 3150-3164.

[22] A. Madeo, S. Gavrilyuk, Propagation of acoustic waves in porous media and their reflection and transmission at a pure-fluid/porous-medium permeable interface, European Journal of Mechanics A-Solids 22 (2010) 897-910.

[23] F.M. Detinko, Lumped damping and stability of beck column with a tip mass, Int. J. Solids. Struct. International Journal of Solids and Structures 40 (2003) 4479-4486.

[24] S. Gutschmidt, O. Gottlieb, Nonlinear dynamic behavior of a microbeam array subject to parametric actuation at low, medium and large dc-voltages, Nonlinear Dynamics 67 (1) (2012) 1-36.

[25] O. Kavianipour, S.H. Sadati, Effects of damping on the linear stability of a freefree beam subjected to follower and transversal forces, Structural Engineering \& Mechanics 33 (2009) 709-724.

[26] H. Ziegler, Die stabilit/itskriterien der elastomechanik, Inženýr architekt 20 (1952) 49-56.

[27] V.V. Bolotin, N.I. Zhinzher, Effects of damping on stability of elastic systems subjected to non-conservative forces, International Journal of Solids and Structures 5 (1969) 965-989.

[28] A.I. R., V.I. Yudovich, The stability of visco-elastic rods, Izvestiya Akademii Nauk SSSR Mekhanika Tverdogo Tela 2 (1974) 78-87.

[29] G.G. Denisov, V.V. Novikov, The stability of a rod loaded by a "follower" force, Izvestiya Akademii Nauk SSSR Mekhanika Tverdogo Tela 1 (1975) $150-154$.

[30] A.R. Seyranian, The destabilization paradox in stability problems for nonconservative systems, Uspekhi Mekhanizatsii 13 (1990) 89-124.

[31] O.N. Kirillov, The destabilization paradox, Doklady Rossijskaya Akademii Nauk 395 (2004) 614-620.

[32] O.N. Kirillov, A.P. Seyranian, The effect of small internal and external damping on the stability of distributed non-conservative systems, Journal of Applied Mathematics and Mechanics 69 (2005) 529-552.

[33] M.A. Langthjem, Y. Sugiyama, Optimum design of cantilevered columns under the combined action of conservative and nonconservative loads: part i: the undamped case, Computers \& Structures 74 (2000) 385-398.

[34] S. Adali, Stability of a rectangular plate under nonconservative and conservative forces, International Journal of Solids and Structures 18 (1982) 1043-1052.

[35] A. Paolone, M. Vasta, A. Luongo, Flexural-torsional bifurcations of a cantilever beam under potential and circulatory forces i: non-linear model and stability analysis, International Journal of Nonlinear Mechanics 41 (4) (2006) 586-594.

[36] A. Paolone, M. Vasta, A. Luongo, Flexural-torsional bifurcations of a cantilever beam under potential and circulatory forces ii: post-critical analysis, International Journal of Nonlinear Mechanics 41 (4) (2006) 595-604.

[37] H. Troger, A. Steindl, Appendix L, in: Nonlinear Stability and Bifurcation Theory, Springer, Wien, New York, 1991, pp. 366-375.

[38] A. Luongo, A. Di Egidio, A. Paolone, Multiple scale bifurcation analysis for finite-dimensional autonomous systems, in: Recent Research Developments in Sound and Vibration, Transworld Research Network, Kerala, India, 2002, pp. 161-201. 
[39] A. Luongo, F. D'Annibale, Bifurcation analysis of damped visco-elastic planar beams under simultaneous gravitational and follower forces, International Journal of Modern Physics B 26 (2012), id. 1246015.

[40] A. Nayfeh, Perturbation Methods, Wiley-VCH, 2000.

[41] A. Luongo, A. Di Egidio, Bifurcation equations through multiple-scale analysis for a continuous model of a planar beam, NonLinear Dynamics 41 (2005) 171-190.

[42] A. Di Egidio, A. Luongo, A. Paolone, Linear and non-linear interactions between static and dynamic bifurcations of damped planar beams, International Joural of Nonlinear Mechanics 42 (2007) 88-98.

[43] A. Luongo, A. Di Egidio, Divergence, hopf and double-zero bifurcations of a nonlinear planar beam, Computers \& Structures 84 (2006) 1596-1605.

[44] S. Alessandroni, U. Andreaus, F. dell'Isola, M. Porfiri, A passive electric controller for multimodal vibrations of thin plates, Computers \& Structures 83 (2005) 1236-1250.
[45] C. Maurini, J. Pouget, F. dell'Isola, On a model of layered piezoelectric beams including transverse stress effect, International Journal of Solids and Structures 41 (2004) 4473-4502.

[46] J. Alibert, P. Seppecher, F. dell'Isola, Truss modular beams with deformation energy depending on higher displacement gradients, Mathematics and Mechanics of Solids 8 (2003) 51-73.

[47] S. Vidoli, F. dell'Isola, Modal coupling in one-dimensional electromechanical structured continua, Acta Mechanica 141 (2000) 37-50.

[48] A. Luongo, F. D'Annibale, Linear stability analysis of multiparameter dynamical systems via a numerical-perturbation approach, AIAA Journal 49 (9) (2011) 2047-2056.

[49] A. Luongo, A. Paolone, A. Di Egidio, Sensitivities and linear stability analysis around a double zero eigenvalue, AIAA Journal 38 (2000) 702-710. 